\title{
Multi-Channel Near-Field Terahertz Communications Using Reprogrammable Graphene-Based Digital Metasurface
}

\author{
Kasra Rouhi, Graduate Student Member, IEEE, Seyed Ehsan Hosseininejad, Sergi Abadal, Member, IEEE, \\ Mohsen Khalily, Senior Member, IEEE, Rahim Tafazolli, Senior Member, IEEE
}

\begin{abstract}
Digital metasurfaces have opened unprecedented ways to accomplish novel electromagnetic devices thanks to their simple manipulation of electromagnetic waves. However, the metasurfaces leveraging phase-only or amplitude-only modulation restricted the full-functionality control of the devices. Herein, a digital graphene-based metasurfaces engineering wavefront amplitude and phase are proposed for the first time to tackle this challenge in the terahertz (THz) band. The concept and its significance are verified using reprogrammable multi-focal metalens based on a 2/2-bit digital unit cell with independent control of 2-bit states of amplitude and phase individually. Moreover, we introduce a novel method to directly transmit digital information over multiple channels via the reprogrammable digital metasurface. Since these metasurfaces are composed of digital building blocks, the digital information can be directly modulated to the metasurface by selecting specific digital sequences and sent them to predetermined receivers distributed in the focal points. Following that, a multi-channel THz high-speed communication system and its application to build three-dimensional wireless agile interconnection are demonstrated. The presented method provides a new architecture for wireless communications without using complicated components of conventional systems. This work motivates versatile meta-devices in many applications envisioned for the THz frequencies, which will play a vital role in modern communications.
\end{abstract}

Index Terms-Graphene, Interconnects, Metasurface, Multifocal Lens, Terahertz Communications.

\section{INTRODUCTION}

$\mathbf{M}$ ETASURFACES are defined as artificial structures that consist of a two-dimensional pattern of sub-wavelength scattering thin particles [1]. Two-dimensional materials are excellent platforms for intriguing physics and optoelectronic applications because of their ultrathin thicknesses and unique

This work was supported by Home of the $5 \mathrm{G}$ and $6 \mathrm{G}$ Innovation Centres. The work of S. Abadal was supported by the European Unions Horizon 2020 Research and Innovation Programme under Grant 863337.

Kasra Rouhi is with the School of Electrical Engineering, Iran University of Science and Technology, Tehran, 1684613114, Iran e-mail: kasrarouhi@elec.iust.ac.ir.

Seyed Ehsan Hosseininejad is with the Department Electrical and Computer Engineering, Babol Noshirvani University of Technology, Babol, Iran; and also with the Institute for Communication Systems (ICS), Home of the 5G and 6G Innovation Centres, University of Surrey, U.K. (email: sehosseininejad@nit.ac.ir).

Sergi Abadal is with NaNoNetworking Center in Catalonia (N3Cat), Universitat Politecnica de Catalunya, 08034 Barcelona, Spain (email: abadal@ac.upc.edu).

Mohsen Khalily and Rahim Tafazolli are with the Institute for Communication Systems (ICS), Home of the 5G and 6G Innovation Centres, University of Surrey, U.K. (email: m.khalily@surrey.ac.uk; r.tafazolli@ surrey.ac.uk). properties in THz and optics [2]. Adjusting the spatial distribution of specified scatterers, a metasurface enables the manipulation of phase, amplitude, and polarization of electromagnetic waves in reflection and transmission modes. They have been designed to control electromagnetic waves in various frequency spectrum bands for specific purposes such as refractive index sensing [3], super diffraction [4], subwavelength imaging [5], beam shaping [6], and signal processing [7].

The lack of tunability is the main drawback in conventional metasurfaces. In response to the limitations of passive metasurfaces, tunable metasurfaces have been proposed [8]. In this class of metasurfaces, reconfigurability has been achieved by employing tunable lumped elements (e.g., varactors and pin diodes) [9], electrostatic biasing [10], microelectromechanical systems (MEMS) [11], thermal tuning [12], and optical excitation [13]. On the other hand, digital metasurfaces are outlined as a new generation of metasurfaces that discretize electromagnetic characteristics of the structure to realize simple wave manipulation with straightforward tunability [14], [15]. The reconfigurablity feature enhances the performance metrics of metasurfaces, which can be programmed globally at the whole meta-structure level or locally at the unit cell level according to required functionality. Graphene is a flat mono-atomic layer of Carbon atoms organized in a twodimensional (2D) honeycomb-like lattice. This 2D material has recently emerged as a powerful plasmonic material that can overcome the drawbacks of metals from terahertz $(\mathrm{THz})$ to mid-infrared regimes [16]. Control of graphene's electric conductivity through external biasing or chemical doping has enabled radically different tunable devices, which are providing significant interest in nanotechnology, biomedical engineering, material science, physics, and green chemistry [17]-[19]. Moreover, Surface Plasmon Polaritons (SPP) waves supported by graphene in the mentioned frequencies results in miniaturization of devices. Consequently, graphene has been conceptually assessed in a myriad of metasurface applications such as absorbers [20], nanoantennas [10], polarization-state modulation [21], and ultrasensitive biosensors [22].

In the context of metasurfaces, as an established method, controlling the reflection/transmission phase of the unit cell allows engineering the wavefront of the scattered field [23], [24]. Recently, simultaneous control over both the amplitude and phase has been introduced in several kinds of research [25]. This extra control over the unit cells enables us to manipulate both far-field and near-field regions fully. 


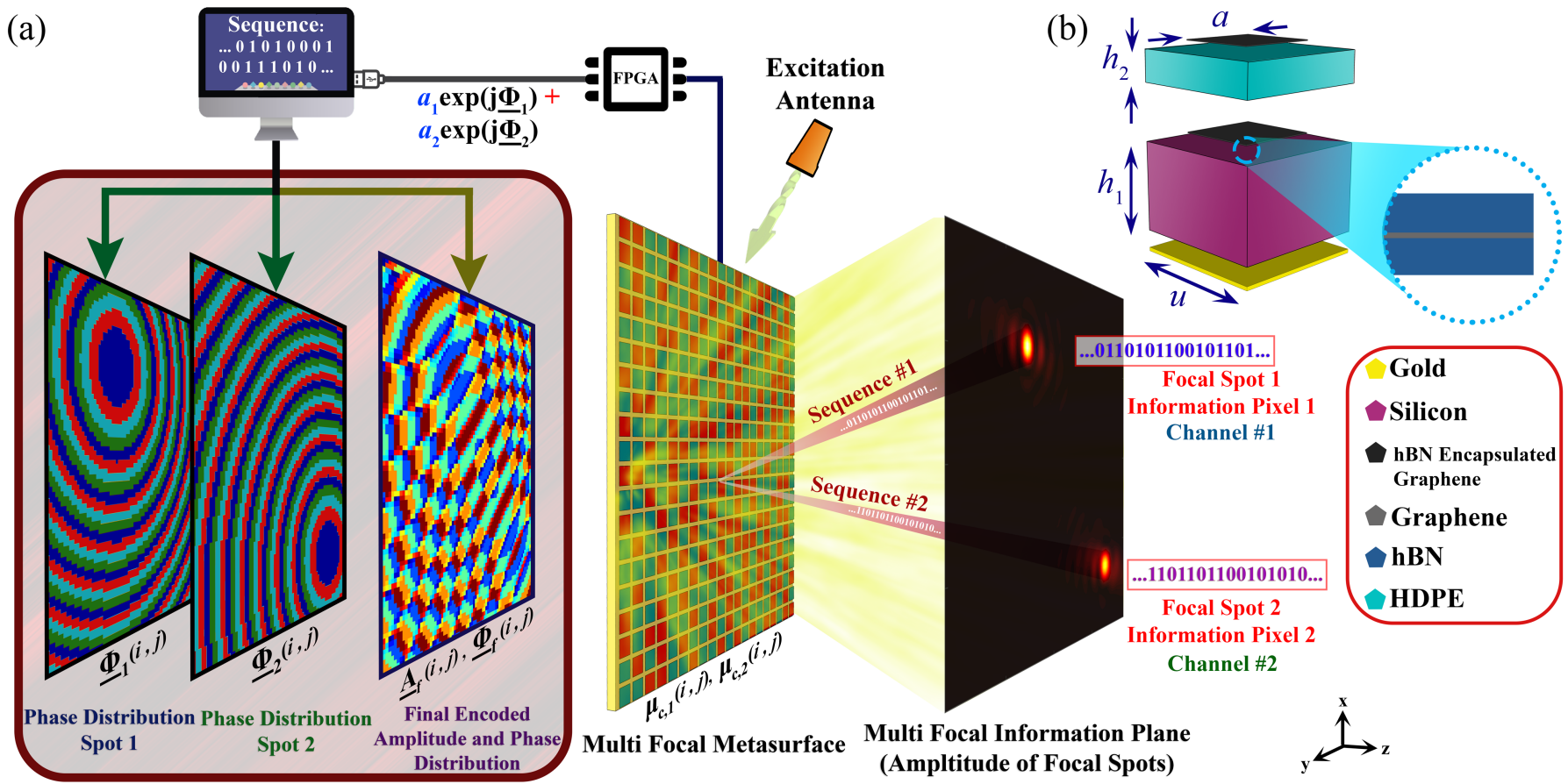

Fig. 1. (a) Schematic view of reprogrammable digital metasurface with complete amplitude and phase engineering for multi-channel THz communication For a bi-focal meta-lens as an illustration, calculating $\underline{\Phi_{1}}$ and $\underline{\Phi_{2}}$ as the phase profiles of two different focal points, $\boldsymbol{A}_{\boldsymbol{f}}$ and $\boldsymbol{\Phi}_{\boldsymbol{f}}$, are respectively calculated as the final amplitude and phase profiles of metasurface using superposition principle. (b) Perspective of the unit cel $\Gamma$ in reprogrammable digital metasurface whose operational status can be dynamically adjusted by proper DC external biasing.

Lianlin $\mathrm{Li}$ et al. used "ON" and "OFF" switches to verify more precise control over the reflected wave's wavefront for holography applications [26]. Furthermore, the digital spacetime codes have been applied on a digital metasurface in the microwave frequencies for multi-channel transmission of information [27]. In conventional wireless communication systems, digital information is modulated by manipulating the amplitude, phase, and polarization of the wave. In the advanced communication architecture, digital sequences will be directly modulated to the metasurface, resulting in different electric field distributions in the near-field [28]. Hence it is possible to use the variation of electric field patterns in the near-field to transmit data through the communication channel. Here, we propose a reprogrammable $\mathrm{THz}$ metasurface design approach based on graphene leveraging both wave amplitude and phase manipulation. The results show that the graphenebased metasurface can bring Fabry-Perot resonance and enhance the localized electric field, making abrupt amplitude and phase changes in the reflected wave. As Fig. 1 shows conceptually, a versatile multi-focal meta-lens is presented to develop an enhanced multi-channel communication link with tunability based on unconventional spatial and temporal modulation.

The remainder of the paper is organized as follows. First, we describe the unit cell design procedure and possible controlling states, including the selected geometry, used materials, biasing method, and fabrication concerns in Section II. Next, the formulation that explains the required digital amplitude and phase distribution of the metasurface for multi-focal focusing is discussed in Section III. Besides, we examine the theoret- ical techniques based on Maxwell's equations to investigate electric field distribution in front of the meta-structure in this section. The theoretical and numerical results of multifocal focusing for different cases are presented in Section IV. Moreover, we will discuss the power control of the focusing spots independently in this section. In order to investigate the proposed structure application, in Section V, it is explained how the multi-focal lens can be applied to design advanced multi-channel transmissions. Finally, the multi-focal metalens is proposed for its application in the context of threedimensional wireless agile interconnects.

\section{Graphene-Based Unit Cell with Digital Amplitude AND Phase CONTROL}

\section{A. Graphene Modeling}

Graphene is an exciting two-dimensional material with particular characteristics in various electromagnetic spectrums. According to the sensitivity of graphene conductivity to external gate bias and the unique frequency dispersion of graphene in $\mathrm{THz}$ band for propagation of surface plasmon waves, this material can be employed to efficiently and dynamically control the THz waves [29], [30]. We modeled graphene as an infinitesimally thin sheet with surface impedance $Z(\omega)=$ $1 / \sigma(\omega)$, where $\sigma(\omega)$ is the frequency dependent complex conductivity of graphene. The complex conductivity is given by [31]

$$
\sigma(\omega)=\frac{2 e^{2}}{\pi \hbar} \frac{k_{B} T}{\hbar} \ln \left[2 \cosh \left[\frac{\mu_{c}}{2 k_{B} T}\right]\right] \frac{i}{\omega+i \tau^{-1}}
$$




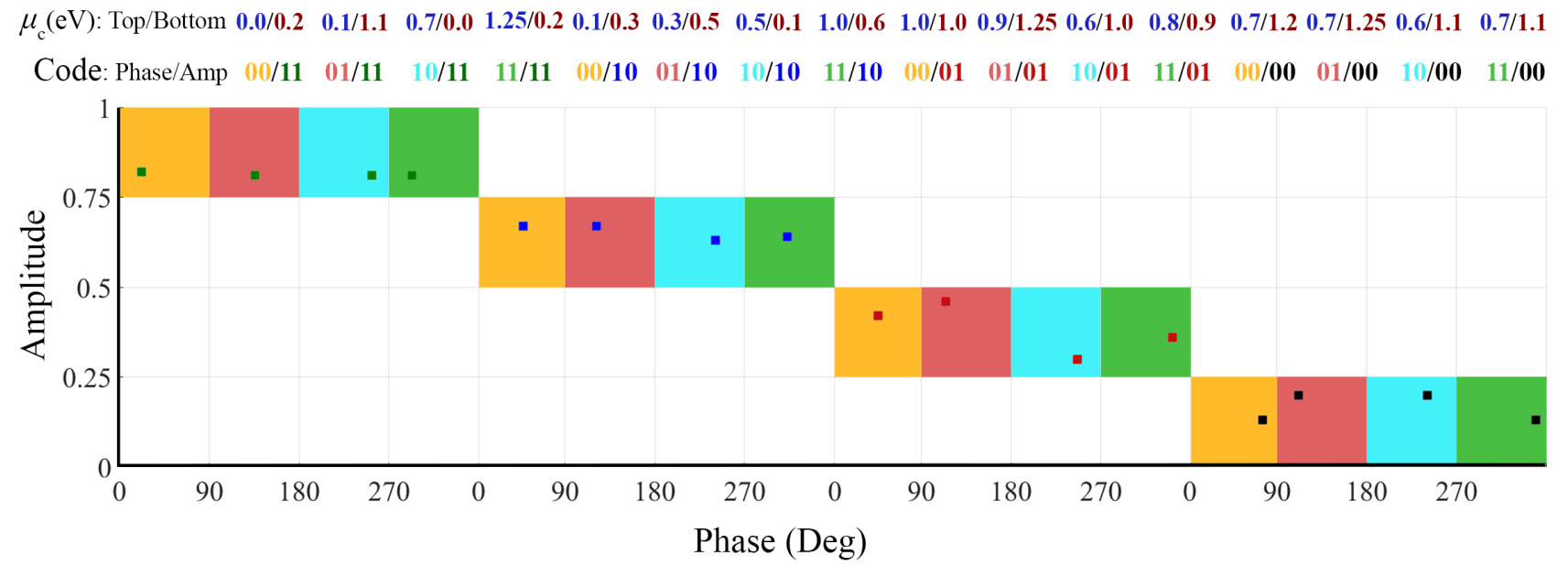

Fig. 2. The phase/amplitude digital states for the 2/2-bit unit cell with changing chemical potentials of top and bottom graphene patches. Each element's color represents the digital amplitude (small dot) and phase (large square). Also, the small dots' position within each large square represents the actual amplitude and phase extracted from the simulated unit cell. The digital code of each phase/amplitude set and chemical potentials pairs is indicated at the top of the figure.

where $e, \hbar$, and $k_{B}$ are constants corresponding to the charge of an electron, the reduced Planck constant, and the Boltzmann constant, respectively [31]. Parameters $T, \tau$, and $\mu_{c}$ correspond to the temperature, the relaxation time, and the chemical potential of the graphene monolayer, respectively. Note that this expression neglects the edge effects of the graphene and considers that the Drude-like intraband contribution dominates, which are experimentally validated assumptions at the sizes and frequencies considered in this work. It is worth noting that here graphene as a two-dimensional material is assumed to be encapsulated with hexagonal Boron Nitride $(\mathrm{hBN})$ for achieving more stability and higher relaxation time. Therefore, we consider a graphene quality leading to a relaxation time of $\tau=0.6 \mathrm{ps}$ which is reasonable by this approach. The individual reflection amplitude and phase control in graphene metasurfaces are obtained via complex conductivity changes when biased by applying the DC voltage to produce a constant electric field. This effect can be modeled through the chemical potential value of $\mu_{c}$. Indeed, the chemical potential can be tuned through electrostatic biasing in a real-time manner. Hence, we can meet the required amplitude and phase layout by controlling external biasing via the highspeed processors.

\section{B. Unit Cell Design and Amplitude/Phase Digital States}

A reflective graphene-based unit cell has been selected as a fundamental element to achieve a meta-lens. The stacking layers of the unit cell from bottom to top are the following, as shown in Figure 1 (b). A silicon substrate with a thickness $h_{1}$ is stacked on a thin gold layer as a metallic layer. A graphene square patch with length $a$ is deployed on this substrate and sandwiched by another substrate on top. The upper substrate is high-density polyethylene (HDPE), with a thickness of $h_{2}$. Finally, another graphene patch is deployed with a similar size of $a$ in the uppermost part of the unit cell. In the proposed structure, the lateral dimension of the unit cell is $u$ in both directions. Also, another thin layer is embedded into the substrates below the graphene patches to make a virtual ground plane for DC biasing. Doped silicon is the right candidate as a virtual ground layer because it conducts DC voltage efficiently [32], [33]. Therefore, an electrostatic gate voltage is applied between the graphene layer and the doped silicon frame to modulate the chemical potential. In the reprogrammable unit cell, the geometric parameters are considered as: $h_{1}=10 \mu \mathrm{m}$, $h_{2}=4 \mu \mathrm{m}, a=12 \mu \mathrm{m}$, and $u=20 \mu \mathrm{m}$.

Current realizations of digital metasurfaces are mostly limited to phase control (e.g., beam steering and power concentration) or amplitude control (e.g., absorption and SLL control). In this paper, we utilize simultaneous manipulation of the reflection amplitude and phase to attain full control over the functionality of the metasurface. In recent studies, graphenebased phase-only metasurface for the phase control is proposed [10], [34]. This case has a useful application in simple farfield and near-field wave engineering, but amplitude control is required for more complex beam synthesis [35]. For this reason, we add more freedom to the structure by considering a 2-bit amplitude discretization as $0.25,0.50,0.75$, and 1 that adds up the encoder to 2/2-bits, ergo 16 states in overall. Consequently, we can achieve more degrees of freedom to control the reflected wave in the target frequency.

Discrete valid states of amplitude and phase responses of the unit cell along with the essential chemical potentials of the two graphene patches and their corresponding digital bits for both amplitude and phase are shown in Figure 2. All the presented results in this figure are based on unit cell simulation by assuming periodic boundary conditions in both $x$ - and $y$ - directions. At the same time, Floquet ports are assigned to the $z$-direction. The operating frequency is $2 \mathrm{THz}$, and all extracted results have been obtained in this frequency. It is worth noting that due to the symmetric geometry of the designed metasurface, this structure is polarization insensitive and has the same behavior under both $x$ - and $y$-polarized illuminations. In addition, a proper circuit model and required value for components are provided for the proposed structure in Appendix A.

The Fabry-Perot interference theory can give a profound 
insight into the underlying physics of the multi-layer metasurface. We can use the electric field distribution and Fabry-Perotlike cavity model to explain the operating mechanisms. When an incident wave illuminates, two Fabry-Perot-like cavities are excited. The illuminated wave can transmit from the front graphene patch and move forth and back between the front and middle graphene patch, forming the first Fabry-Perot-like cavity. Then the transmitted wave travels forth and back in the second Fabry-Perot-like cavity formed by the middle graphene patch, and the back metallic ground plane [36]. A typical Fabry-Perot resonance associated with an amplitude and phase change is observed depending on the constant thickness of the substrates and variable chemical potential of both graphene patches [37].

\section{Biasing Voltage Calculation}

The employed biasing configuration of the proposed unit cell is in Figure 1, where the DC voltage is applied to the graphene patches separately by an ultra-thin layer $\left(h_{A l}\right.$ $=65 \mathrm{~nm}$ ) of $\mathrm{Al}_{2} \mathrm{O}_{3}$ with the permittivity of $\varepsilon_{A l}=9.9$. Such a biasing structure drastically facilitates the manipulation amplitude and phase reflections. Due to the infinitesimally small thickness of the auxiliary layers, we have neglected them in the schematic figure. For an isolated graphene sheet, the chemical potential is determined by the carrier density [38]

$$
n_{s}=\frac{2}{\pi \hbar^{2} v_{F}^{2}} \int_{0}^{\infty} \epsilon\left(f_{d}(\epsilon)-f_{d}\left(\epsilon+2 \mu_{c}\right)\right) d \epsilon .
$$

Here $n_{s}$ denotes the graphene carrier density, $v_{F}$ refers to the Fermi velocity, and $\epsilon$ is energy. Also, the Fermi-Dirac distribution is determined as

$$
f_{d}(\epsilon)=\left(e^{\left(\epsilon-\mu_{c}\right) / k_{B} T}+1\right)^{-1} .
$$

A simple representation of Equation (2) reads $n_{s}=$ $\left(\varepsilon_{0} \varepsilon_{A l} V_{D C}\right) /\left(h_{A l} e\right)$ in which $V_{D C}$ stands for the required biasing voltage. Hence, the chemical potential of the graphene sheet can be calculated by using a simple capacitor model [39]

$$
\mu_{c}=\hbar v_{F} \sqrt{\pi n_{s}} .
$$

Accordingly, each graphene patch's chemical potential can be dynamically tuned by adjusting the applied DC voltage. The connection between the chemical potential of the graphene patches and the gate voltage can be defined as [40]

$$
V_{D C}=\frac{\mu_{c}^{2} h_{A l} e}{\hbar^{2} v_{F}^{2} \pi \varepsilon_{0} \varepsilon_{A l}} .
$$

Recently, several attempts have resulted in engineering the Fermi velocity by the strength of electron-electron interactions when the environment embedding graphene is modified [41]. In the case of weak electron-electron interactions, the Fermi velocity is expected to be as low as $0.85 \times 10^{6} \mathrm{~m} / \mathrm{s}$, whereas, for strong interactions, the Fermi velocity is as high as $1.73 \times 10^{6}$ $\mathrm{m} / \mathrm{s}$ in suspended graphene [41]. In this paper, relying on the crucial role of dielectric screening in graphene, we assume $v_{F}=1.49 \times 10^{6} \mathrm{~m} / \mathrm{s}$ by using the $\mathrm{hBN}$ dielectric environment [41]. Hence, the biasing electric field using DC voltage is needed to design each of the digital graphene-based unit cells. All of the required DC voltages fall below the breakdown voltage, computed as $65 \mathrm{~V}$ by the I-V curve analysis presented in [42]. This breakdown voltage is corresponding to the breakdown field of $1 \mathrm{~V} / \mathrm{nm}$ [43]. In addition, the required external voltage for biasing graphene layers related to the values employed in this paper is calculated and presented in Appendix B. The required explanation for the potential fabrication method and communication modulation speed is provided in Appendix C and Appendix D.

\section{Multi-Focal Meta-Lens with Amplitude AND PHASE ENGINEERING}

The purpose of this section is to explore the required amplitude and phase profiles for concentrating the reflected wave into multiple arbitrary points. To calculate the amplitude and phase layouts of the metasurface, we first explain the mathematical formulation to achieve a meta-lens with a single arbitrary focal point. Next, we will extend the proposed formulation to a multi-focal function. At last, we will introduce the theoretical approach for calculating near-field distribution. The theoretical method will use to validate the simulation results in Section IV.

\section{A. Formulations of Amplitude and Phase Profiles Across Multi-Focal Meta-Lens}

Without loss of generality, let us first consider a metasurface array illuminated by a normally incident plane wave. In order to focus the energy in an arbitrary focal point defined by $\left(x_{f}, y_{f}, z_{f}\right) \equiv\left(R_{f}, \theta_{f}, \varphi_{f}\right)$, the phase profile across the metasurface $\underline{\boldsymbol{\Phi}}(i, j)$ must fulfill the following condition extracted from the generalized Snell's law of reflection [44]

$$
\begin{aligned}
\underline{\boldsymbol{\Phi}}(i, j)= & k_{0}\left\{\left[\left(R_{f} \cos \theta_{f}\right)^{2}+\left(x_{i}-R_{f} \sin \theta_{f} \cos \varphi_{f}\right)^{2}+\right.\right. \\
& \left.\left.\left(y_{j}-R_{f} \sin \theta_{f} \sin \varphi_{f}\right)^{2}\right]^{1 / 2}-R_{f} \cos \theta_{f},\right\},
\end{aligned}
$$

where $k_{0}$ is the incident beam wavenumber in a vacuum, $x_{i}$, and $y_{j}$ are the coordinates of the point on the metasurface. A multi-focal functionality is achieved by combining the several phase-only concentration layouts with the help of the superposition principle [15]. It is seen that after the superposition of arbitrary phases, we will end up with a complex number, including both amplitude and phase. The required amplitude and phase distributions over the metasurface for multi concentration points are calculated as [45]

$$
\sum_{n=1}^{N} a_{n} \cdot e^{-j \underline{\boldsymbol{\Phi}}_{n}}(i, j)=\underline{A}_{f}(i, j) e^{-j \underline{\boldsymbol{\Phi}}_{f}(i, j)},
$$

where $\underline{\Phi}_{\boldsymbol{n}}$ is the phase profile, $a_{n}$ is the weight factor (amplitude coefficient) of $n$-th focal point, and $\mathrm{N}$ indicates the number of focus spots. Also, $A_{f}$ is the final amplitude profile, and $\boldsymbol{\Phi}_{\boldsymbol{f}}$ is the final phase profile of the multi-focal meta-lens. Consequently, by employing amplitude and phase 
(a)

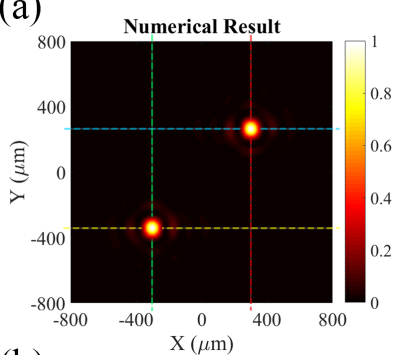

(b)

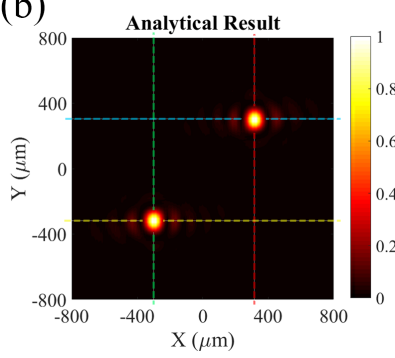

(c)

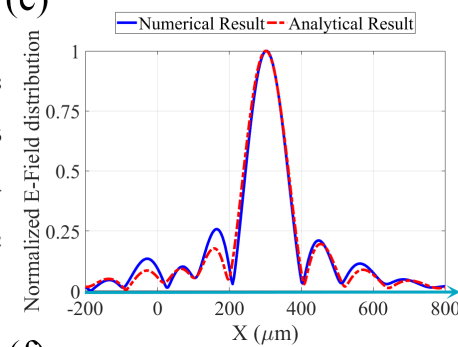

(f)

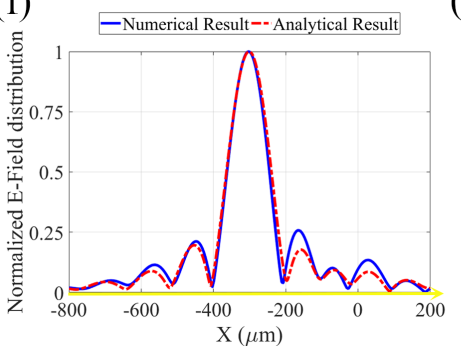

(d)

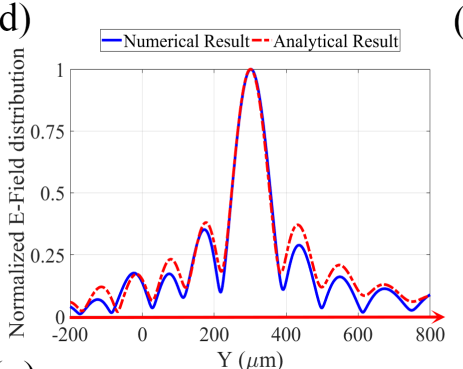

(g)

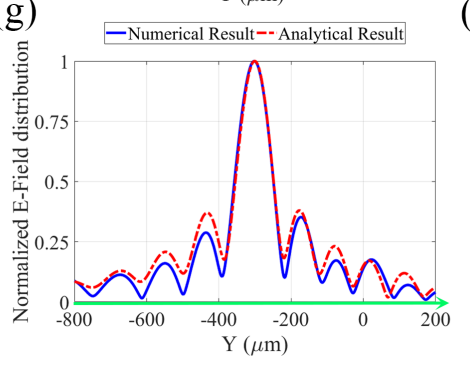

(e)

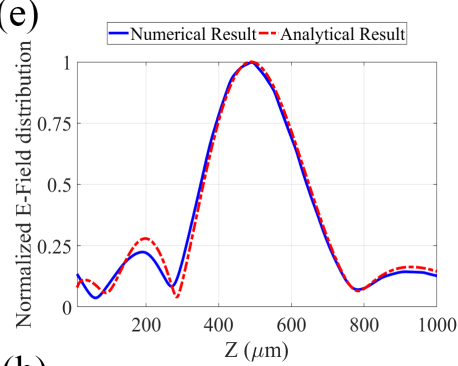

(h)

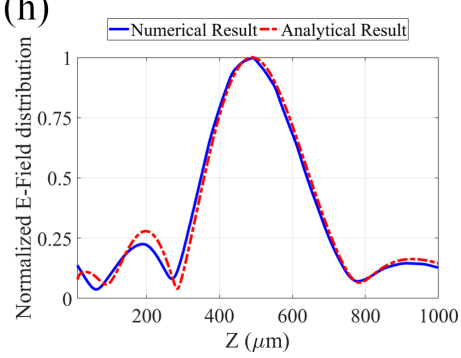

Fig. 3. (a), (b) Two-dimensional electric field distributions at the focal plane. (c)-(h) One-dimensional electric field patterns of graphene-based meta-lens with the foci coordinates as $x_{1}=300 \mu \mathrm{m}, y_{1}=300 \mu \mathrm{m}, z_{1}=500 \mu \mathrm{m}$, and $x_{2}=-300 \mu \mathrm{m}, y_{2}=-300 \mu \mathrm{m}, z_{2}=500 \mu \mathrm{m}$. These figures contain the numerical and analytical results extracted from the full-wave simulations and the theoretical calculations.

simultaneously, we can attain a full engineered field pattern that was not possible only with phase control of the unit cells. Note that dividing metasurface to multiple phase-only modulation metasurface enables multi-focal arrangements, but at the cost of increasing the aperture size and also limiting the achievable focal points positions [12].

\section{B. Theoretical Calculations of Near-Field Distribution}

After calculating the amplitude and phase profiles of reflective elements across the metasurface, here the formulation is developed to assess the metasurface response. Applying the Huygens principle and assuming each unit cell as a secondary small electrical source, we can calculate the scattered electromagnetic fields. Consider an $x$-polarized incident plane wave that excites an $x$-directed current density $J_{x}$ on the unit cells. Therefore, the inhomogeneous potential wave equation is denoted by

$$
\nabla^{2} A_{x}+k^{2} A_{x}=-\mu_{0} J_{x}\left(x^{\prime}, y^{\prime}\right)
$$

where $A_{x}$ is the vector magnetic potential, $k$ is the wave number and $\mu_{0}$ is the vacuum permeability. Since the unit cells are electrically small, it is reasonable to assume each unit cell as a punctual source. Consequently, the Green's function for the wave equation allows us to write the solution of Equation (8) as

$$
A_{x}=\frac{\mu_{0}}{4 \pi} J_{x}\left(x^{\prime}, y^{\prime}\right) \frac{e^{-j k\left|\mathbf{r}-\mathbf{r}^{\prime}\right|}}{\left|\mathbf{r}-\mathbf{r}^{\prime}\right|}
$$

In the above equation, $\mathbf{r}=(x, y, z)$ and $\mathbf{r}^{\prime}=\left(x^{\prime}, y^{\prime}\right)$ are the vector positions of the observation and source points, respectively. In this case, source points are the center of unit cell positions, and the observation points are the arbitrary number of points located on the near-field observation plane. Moreover, the distance between a certain unit cell and an observation point is written as

$$
\left|\mathbf{r}-\mathbf{r}^{\prime}\right|=\sqrt{\left(x-\left(i-\frac{1}{2}\right) u\right)^{2}+\left(y-\left(j-\frac{1}{2}\right) u\right)^{2}+z^{2}},
$$

where $i$ and $j$ are the unit cell pointers, $u$ is the unit cell size. The magnetic and electric fields can be found by applying $H=\frac{1}{\mu_{0}} \nabla \times A$ and $E=\frac{1}{j \omega \varepsilon_{0}} \nabla \times H$, respectively. This allows to obtain a closed form expression for the electric field as

$$
\begin{aligned}
E_{x}(x, y, z) & =\frac{-j \eta}{4 \pi k}\left(G_{1}+\left(x-x^{\prime}\right)^{2} G_{2}\right) J_{x} e^{-j k\left|\mathbf{r}-\mathbf{r}^{\prime}\right|}, \\
G_{1} & =\frac{-1-j k\left|\mathbf{r}-\mathbf{r}^{\prime}\right|+k^{2}\left|\mathbf{r}-\mathbf{r}^{\prime}\right|^{2}}{\left|\mathbf{r}-\mathbf{r}^{\prime}\right|^{3}}, \\
G_{2} & =\frac{3+j 3 k\left|\mathbf{r}-\mathbf{r}^{\prime}\right|-k^{2}\left|\mathbf{r}-\mathbf{r}^{\prime}\right|^{2}}{\left|\mathbf{r}-\mathbf{r}^{\prime}\right|^{5}}
\end{aligned}
$$

In the above equation, $\eta=120 \pi$ is the impedance of free space. Finally, the electric field distribution in front of the metasurface is obtained by adding the contribution of its $M \times$ $M$ unit cells as

$$
\begin{array}{r}
E_{x}(x, y, z)=\frac{-j \eta}{4 \pi k} \sum_{i=1}^{M} \sum_{j=1}^{M}\left(G_{1}+\left(x-x^{\prime}\right)^{2} G_{2}\right) \\
\exp \left[j\left(\underline{\Phi}(i, j)-k\left|\mathbf{r}-\mathbf{r}^{\prime}\right|\right)\right] .
\end{array}
$$

\section{RESULTS AND DisCUSSION}

This section will investigate the advanced approach to design assorted multi-focal samples at various points in the whole upper half-space. Then, we will simulate the corresponding layout using CST Studio Suite and calculate the theoretical electric field distribution for the samples. Finally, we will compare the numerical and analytical results and discuss independent power control of multiple focus points as an engrossing digital amplitude and phase metasurfaces capability. 
Before demonstrating the methodology, it is emphasized that continuous profiles of amplitude and phase will be obtained using the analytical formulation mentioned in Equation (7). Depending on the available bits, we face a limited number of states for unit cell amplitude and phase. In our case, we account for 2-bit for amplitude and 2-bit for phase, making it a 4-bit digital state overall. Consequently, the continuous amplitude and phase quantities are transformed into 16 discrete states with the specific amplitude and phase. As a matter of fact, the actual amplitude and phase profiles of the digital metasurface are extracted by spatially discretizing $A_{f}$ and $\boldsymbol{\Phi}_{\boldsymbol{f}}$, and rounding off the values to those offered by the closest digital states (see Figure 2).

\section{A. Bi-focal Concentration}

In order to represent the methodology, assume that $N$ $=2$ and $M=80$, and we attempt to concentrate the energy around two foci simultaneously using a reprogrammable metasurface. In the presented examples in this subsection, we assign equal power to both focal points, so $a_{1}=0.5$ and $a_{2}=0.5$. In the next subsections, we will investigate the inequality on the output power distributions of two foci. According to the conventional method, we have two foci by applying two different phase profiles, but it is not simultaneous multi-focusing. So, we combine these two phase layouts by employing the superposition theory as mentioned in Equation (7). As first example, consider two uncorrelated foci being at $x_{1}=300 \mu \mathrm{m}, y_{1}=300 \mu \mathrm{m}, z_{1}=500 \mu \mathrm{m}$, and $x_{2}=-300 \mu \mathrm{m}$, $y_{2}=-300 \mu \mathrm{m}, z_{2}=500 \mu \mathrm{m}$ positions. Both the focal points have been placed in the same plane, which means they have a similar position in the $z$-direction. Regarding Equation (7), after a combination of two obtained phasors, we must end up with a complex profile, including amplitude and phase. A multi-focusing digital device is implemented if we discretize both amplitude and phase and utilize the corresponding unit cell state for each one. Figure 3 exhibits the results extracted by the numerical and analytical approaches. All the results in this figure are represented at unit cell operating frequency. The presented two-dimensional electric field distribution makes a qualitative sense of power focusing and concentration accuracy in the predetermined focus points. Also, the one-dimensional electric field distributions versus $x$-, $y$ - and $z$-axes are compared in this figure. Figures 3(c)-(e) and Figures 3(f)-(h) show calculated results for first and second focal points respectively. First, the numerical and analytical electric field distributions are in excellent agreement. Second, by applying digital amplitude and phase engineering via proper external biasing, two focal points are simultaneously appeared with one single digital metasurface accurately.

As a second scenario, we have selected the two foci with two different focal depths (two focal points along the $z$-axis) $x_{1}=0 \mu \mathrm{m}, y_{1}=0 \mu \mathrm{m}, z_{1}=450 \mu \mathrm{m}$, and $x_{1}=0 \mu \mathrm{m}$, $y_{1}=0 \mu \mathrm{m}, z_{2}=1350 \mu \mathrm{m}$. As studied earlier, the single digital metasurface can rearrange to redirect the beam to different points. It is only necessary to calculate the required amplitude and phase and then translate it to the needed chemical potential. Then we estimate the DC voltage value
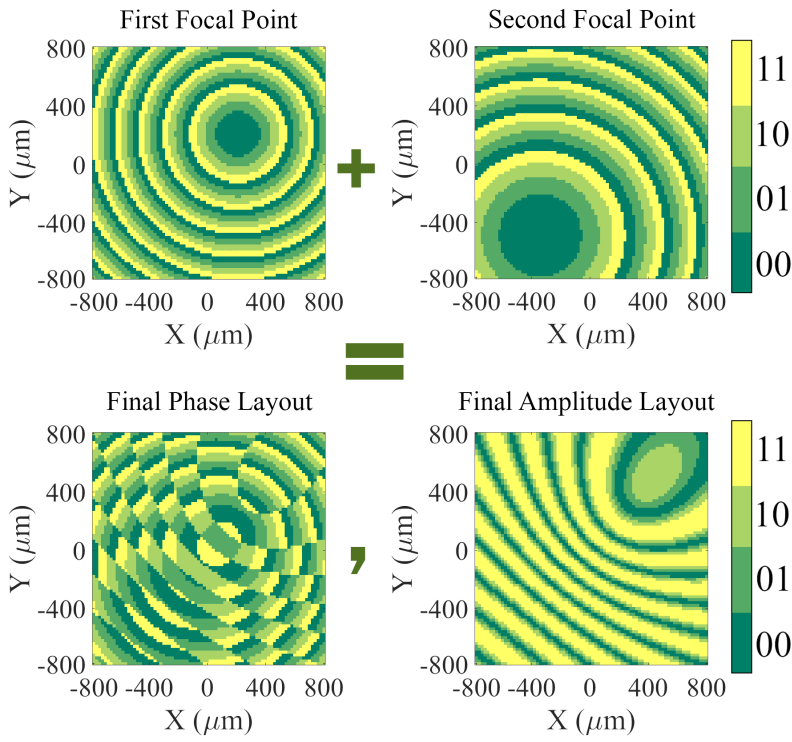

Fig. 4. Two figures in the first row show the required digital phase profile used to achieve the individual focal points at $x_{1}=200 \mu \mathrm{m}, y_{1}=200 \mu \mathrm{m}$, $z_{1}=300 \mu \mathrm{m}$, and $x_{2}=-350 \mu \mathrm{m}, y_{2}=-500 \mu \mathrm{m}, z_{2}=1050 \mu \mathrm{m}$. Two figures in the second row exhibit the digital amplitude and phase layouts required to have a multi-focal power concentration on the points mentioned above by using the superposition method.

and apply the DC bias easily by using field-programmable gate array (FPGA). In contrast to the previous example, these two points have been placed in two different planes. In this case, the normalized electric field distributions versus $x$-, $y$ - and $z$ axis are observed in Appendix E and verified the accuracy of the approach.

In the third example, the study is replicated with two concentration spots on entirely different planes and various axes to ensure the generality of method. Indeed, we make sure there is no limitation over the focal point positions. Accordingly, the two distinct focal points are selected at $x_{1}=200 \mu \mathrm{m}, y_{1}=200 \mu \mathrm{m}, z_{1}=300 \mu \mathrm{m}$, and $x_{2}=-350 \mu \mathrm{m}$, $y_{2}=-500 \mu \mathrm{m}, z_{2}=1050 \mu \mathrm{m}$. The required encoded phase layout for each focal point and final amplitude and phase map are illustrated in Figure 4. This figure reveals the approach overview for generating more than one focal point. Furthermore, we plot the normalized electric field distributions versus the $x$-, $y$ - and $z$-axes in Appendix $\mathrm{E}$. There is a close agreement between the numerical and analytical results, which implies that the discretization error incurred by the 16 states has a small impact on the performance of the meta-lens.

The near-field region is the region right next to the metasurface. This region is defined in the range of $2 D^{2} / \lambda$, where $D$ is the maximum linear dimension of the meta-lens, and $\lambda$ is the wavelength of the electromagnetic wave in the operating frequency. According to the demonstrated formulation for near-field, all the presented examples in this paper are located in this region. Also, the far-field region is the region that comes after the near-field region, and the electromagnetic fields are dominated by radiating fields in this region. In this subsection, all examples contain two different focal points, but in the general case, our method shows an excellent capability to make more than two concentration spots in the half-space. 

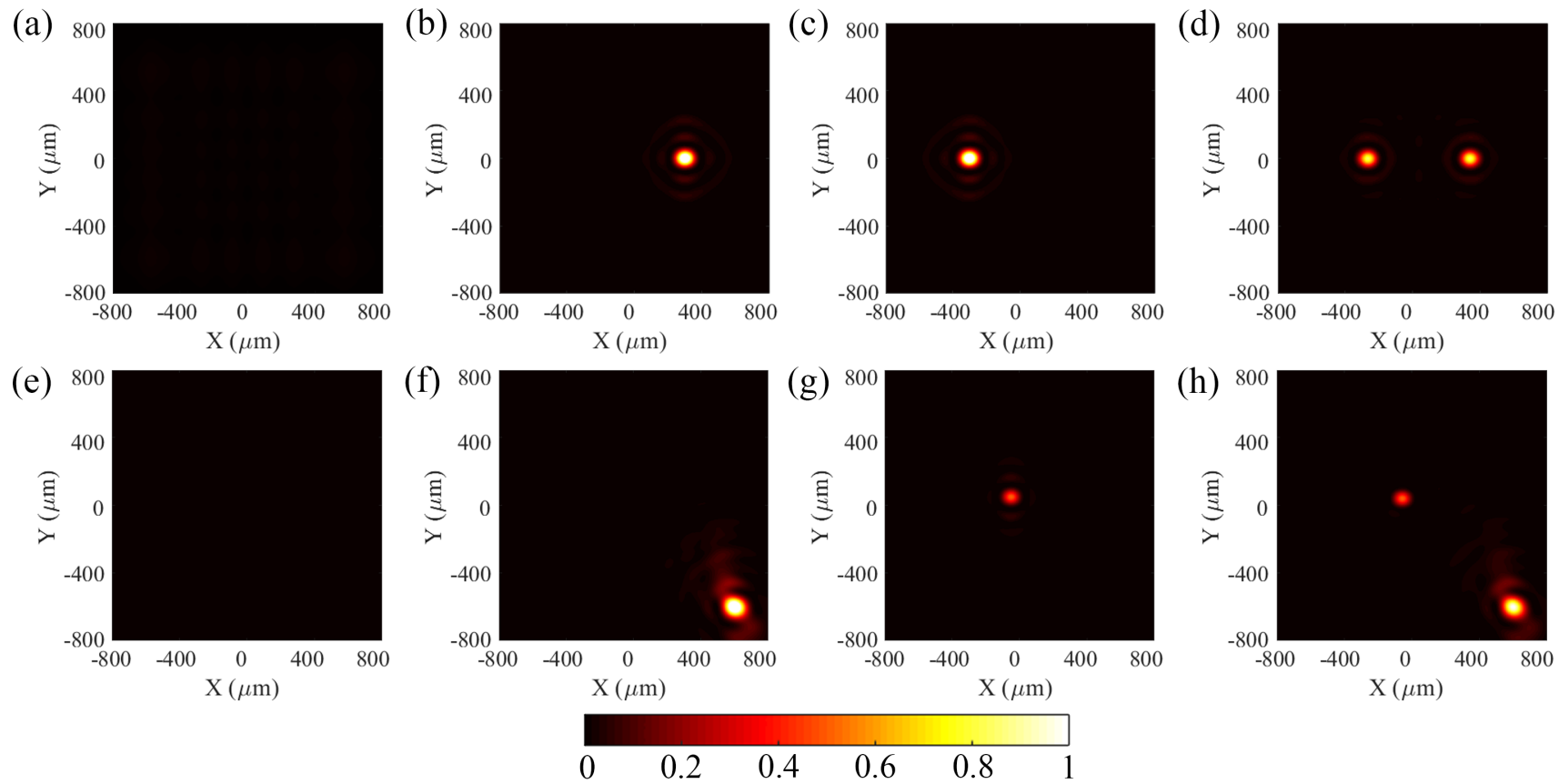

Fig. 5. Simulated power distributions of the tunable bi-focal meta-lens for the combinations of symbols (00, 01, 10, and 11$)$ in the two channels with positions (a)-(d) $x_{1}=-300 \mu \mathrm{m}, y_{1}=0 \mu \mathrm{m}, z_{1}=500 \mu \mathrm{m}, x_{2}=300 \mu \mathrm{m}, y_{2}=0 \mu \mathrm{m}, z_{2}=500 \mu \mathrm{m}$ for symmetric case, and (e)-(h) $x_{1}=-50 \mu \mathrm{m}, y_{1}=50 \mu \mathrm{m}$, $z_{1}=400 \mu \mathrm{m}, x_{2}=600 \mu \mathrm{m}, y_{2}=-600 \mu \mathrm{m}, z_{2}=400 \mu \mathrm{m}$ for asymmetric case. (a), (e) Digital information 00, (b), (f) Digital information 01, (c), (g) Digital information 10, and (d), (h) Digital information 11.

\section{B. Power Modification}

In the previous subsection, we have not investigated the power ratios at different focal points. As a matter of fact, the proportion of concentrated power in the desired positions was out of control because we have assumed equal weight factors $a_{n}$ in Equation (7). In this subsection, we will pursue another capability of the digital amplitude and phase metasurface. The proposed meta-device has an exceptional potential to control the concentration power of each focal point individually.

In this subsection, we will study the effect of different weight factors on the concentrated power ratio in the focal points using the generalized Equation (7). Without loss of generality and only for better presentation and comparison, we assume two focal points on the $z$-axis. Two foci are chosen as $x_{1}=0 \mu \mathrm{m}, y_{1}=0 \mu \mathrm{m}, z_{1}=250 \mu \mathrm{m}$, and $x_{2}=0 \mu \mathrm{m}$, $y_{2}=0 \mu \mathrm{m}, z_{2}=800 \mu \mathrm{m}$. Considering the values of the weight factors $\left(a_{1}, a_{2}\right)$ as $(0.2,0.8),(0.3,0.7),(0.4,0.6),(0.5,0.5)$, $(0.6,0.4),(0.7,0.3)$, and $(0.8,0.2)$. Then required amplitude and phase map is calculated. Fig. 12(a) in Appendix F shows the normalized electric field distributions for various weight factors of $a_{1}$ and $a_{2}$ in the $z$-direction. It is observed that the ratio of power in the focal points is adjustable. Consequently, the power ratio of foci is adjustable by changing the digital amplitude and phase layouts via the external DC bias. This functionality has outstanding potential for the applications that need a specified power division. For instance, if two receivers are placed at two distinct points in front of the metasurface with the goal of multi-channel communication, the farther receiver needs higher power than the closer receiver to transmit data more efficiently.

Another capability of the proposed multi-focal metasurface is power attenuation. By controlling the reflection amplitude of the whole structure altogether, we can alternate the power attenuation. For the sake of brevity, we decrease the weight factors $\left(a_{1}, a_{2}\right)$ simultaneously with constant steps as $(0.5$, $0.5),(0.4,0.4),(0.3,0.3)$, and $(0.2,0.2)$ while the same focal points as last example are assumed. The results of electric field distribution is depicted in Fig. 12(b) in Appendix F. The results confirm the power decrement, which is based on power dissipation, not power diffusion.

\section{Arbitrary Power Pattern Synthesis in General Form}

In this part, the capabilities mentioned earlier are employed to design power patterns in arbitrary points in the space. So, two general examples are examined. In first example, we consider two foci at $x_{1}=300 \mu \mathrm{m}, y_{1}=300 \mu \mathrm{m}, z_{1}=500 \mu \mathrm{m}$, and $x_{2}=-300 \mu \mathrm{m}, y_{2}=-300 \mu \mathrm{m}, z_{2}=500 \mu \mathrm{m}$. Unlike the previous examples, in this case, the goal is to send different amounts of power into different foci. The electric field ratio is assumed to be $3: 2$, which is equal to 9:4 ratio in the power pattern. As a result, in this case where the focus points are symmetrically selected, $a_{1}=0.6$ and $a_{2}=0.4$ are chosen as the weight factors. The results for this example are shown in Appendix G. In Fig. 13(a), the two-dimensional power pattern is presented, while in Figs. 13(b) and (c), one-dimensional results are illustrated from two various side views. It is seen that the concentrated power ratio of 9:4 is successfully attained with high efficiency.

In the second example, we examine the metasurface performance in asymmetric power transmission missions. Hence, two foci are positioned at $x_{1}=200 \mu \mathrm{m}, y_{1}=200 \mu \mathrm{m}, z_{1}=$ $300 \mu \mathrm{m}$, and $x_{2}=-350 \mu \mathrm{m}, y_{2}=-500 \mu \mathrm{m}, z_{2}=1050 \mu \mathrm{m}$. in this scenario, the calculation of weight factors is more complicated because the points are not symmetrically selected. Additionally, the focal points are located on different planes, 
and as mentioned earlier, the amount of delivered power decreases as we move away from the metasurface. Consequently, in this case, an optimization method is applied to achieve the required weight factors using the design Equation (7) and the field analysis Equation (11). For instance, in this asymmetric case, we suppose the power ratio to be equal. Based on the extracted results of optimization, the weight factors are $a_{1}=$ 0.42 and $a_{2}=0.58$. Finally, the power patterns are illustrated in Appendix $\mathrm{G}$ for two different focal planes.

\section{Multi-Focal Lens in Communication APPLICATIONS}

The proposed metasurface in this paper has an outstanding capability for wireless communication. It comes to a significant question: Can the electric field distribution be employed as an extra dimension in a metasurface system to transmit digital information to receivers? The answer is yes; in this section, we study the structure capability in two communication scenarios. As observed in the following two subsections, the reprogrammable graphene-based metasurface has an exceptional ability to transmit data at high speed in modern $\mathrm{THz}$ communication systems.

\section{A. Reprogrammable Multi-Channel Near-Field THz Commu- nications with Simultaneous Spatial and Temporal Modula- tions}

By using the graphene-based metasurface, here we build up a new architecture for $\mathrm{THz}$ wireless communications. The digital information, displayed in the form of binary digital sequence, is first encoded on the metasurface via an external processor, i.e., FPGA, and then transmitted to space directly by the information metasurface in the form of electromagnetic waves under the illumination of feed antenna (see Figure 1). Because the generated field in the information plane is dependent on the digital coding sequence, we use multiple receivers located at the data pixel to capture the transmitted digital information by reading the electric field amplitude. In this system, the complicated components in the conventional communication system are removed fundamentally. Then digital information can be perfectly retrieved from the field distribution of the information plane instead of a single point. Also, secure communication and cryptography can be embedded inside the metasurface-based system. Unlike the conventional approach where the transmitted information is encrypted by software, the digital information sent by the graphene-based digital metasurface is fully protected from interception at data pixels from the physical level without using the software encryption.

The digital information sequence is firstly divided into multiple fragments of n-bit binary code. Each digital information is then mapped to the set of 2-bit amplitude and phase code designed by multi-focal formulation presented in Equation (7). Then under the plane wave illumination of the feeding antenna, the digital metasurface will redirect the digital information toward the information plane in the form of an electric field pattern. Then the digital information encoded in the digital sequence can be transmitted by fast changes in the graphene
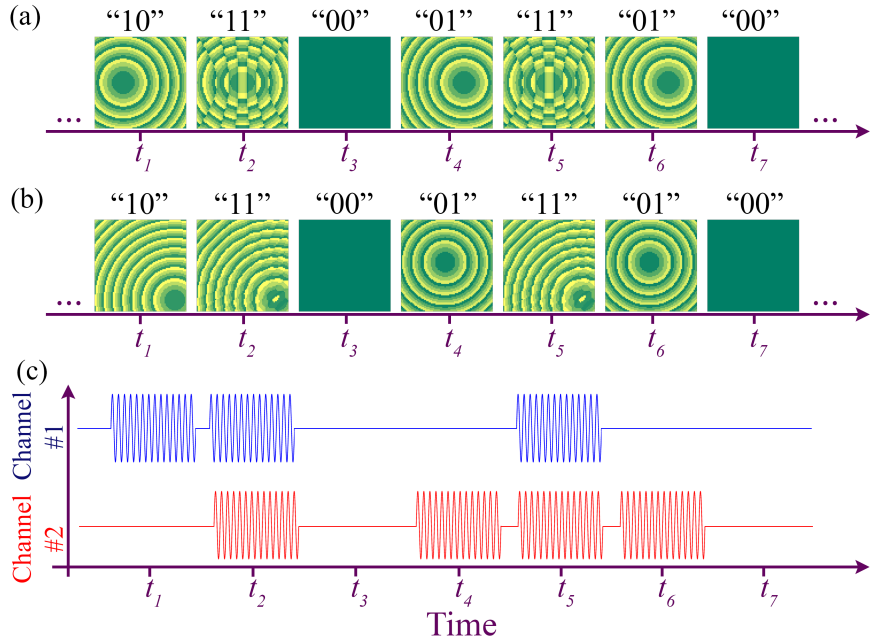

Fig. 6. The digital profiles of the tunable bi-focal meta-lens with the bi-focal points of (a) $x_{1}=-300 \mu \mathrm{m}, y_{1}=0 \mu \mathrm{m}, z_{1}=500 \mu \mathrm{m}, x_{2}=300 \mu \mathrm{m}, y_{2}=$ $0 \mu \mathrm{m}, z_{2}=500 \mu \mathrm{m}$ for symmetric case, and (b) $x_{1}=-50 \mu \mathrm{m}, y_{1}=50 \mu \mathrm{m}$, $z_{1}=400 \mu \mathrm{m}, x_{2}=600 \mu \mathrm{m}, y_{2}=-600 \mu \mathrm{m}, z_{2}=400 \mu \mathrm{m}$ for asymmetric case, to transmit the sequences of $(1,1,0,0,1,0,0)$ and $(0,1,0,1,1$, $1,0)$ over the channel \#1 and channel \#2, respectively. (c) The equivalent time-domain modulated waveform which sent over two parallel channels.

layer's electrostatic bias. Since each amplitude and phase layout determined by the multi-focal equation corresponds to a unique electric field distribution, the transmitted digital information can be recovered by making one to one mapping between the digital information and extracted electric field distribution. In the proposed architecture, two receivers are located in the predetermined data pixels to capture the radiated pattern and electric field amplitude, as shown in Figure 1.

Following Section IV-B, here, a near-field THz communication system with reprogrammable dual channels is developed as a compelling application for the bi-focal meta-lens (right part of Figure 1). So far, it has been demonstrated that power of each focal point is independently modulated in the space by varying the digital aperture codes, including amplitude and phase. In this scenario, the proposed metadevice is employed as a dual channel communication system with both spatial and temporal modulations. By configuring the digital elements, several focal points can be constructed in the near-field region, whose intensity can be dynamically and independently modulated. Hence, the intensity controllable meta-device can perform spatial and temporal modulation simultaneously. This service is used to realize a direct multichannel communication to transmit more than one information sequence concurrently. In the simple case, we considered two focal points that construct two parallel channels, as shown in Figure 1. Two receivers are assumed to be located at the foci. Independent binary digital symbols defined by power amount are transmitted through the two parallel channels. In this system, low intensity in each focal point is considered as a symbol "0", and high intensity is considered as a symbol "1". Accordingly, binary digital information can be transmitted through these channels and by employing predetermined symbols.

To demonstrate the performance of the proposed architecture, two sets of bi-focal points are chosen as $x_{1}=-300 \mu \mathrm{m}$, 
(a)
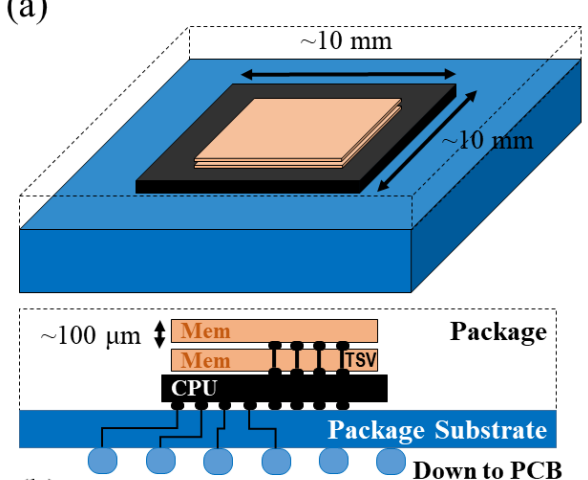

(b) (c) Processor or Router
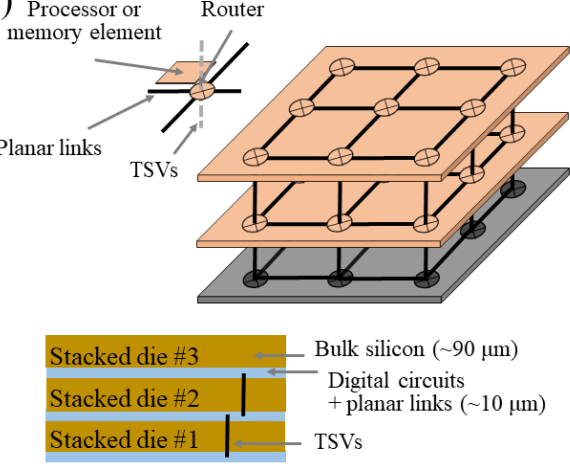

(d)
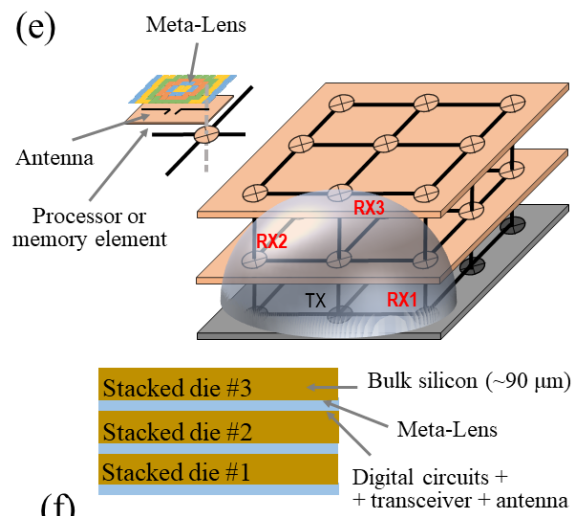

Fig. 7. Schematic representation of the proposed scenario with an agile wireless interconnect enabled by a reprogrammable meta-lens. Figures not to scale. (a), (b) Isometric and cross-section view of the 3D stacked chip within a conventional computing package. (c), (d) Isometric and cross-section illustration of a conventional 3D NoC. (e), (f) Isometric and cross-section illustration of a 3D NoC augmented with antennas and meta-lenses at the processing element level, together with the near-field range of a hypothetical transmitter.

$y_{1}=0 \mu \mathrm{m}, z_{1}=500 \mu \mathrm{m}$, and $x_{2}=300 \mu \mathrm{m}, y_{2}=0 \mu \mathrm{m}$, $z_{2}=500 \mu \mathrm{m}$ for symmetric case and $x_{1}=-50 \mu \mathrm{m}$, $y_{1}=50 \mu \mathrm{m}, z_{1}=400 \mu \mathrm{m}, x_{2}=600 \mu \mathrm{m}, y_{2}=-600 \mu \mathrm{m}$, $z_{2}=400 \mu \mathrm{m}$ for asymmetric receiver location. The simulated power distributions of the symmetric and asymmetric 2-bit combinations are presented in Figure 5. The required amplitude and phase layouts and analytical power distributions have been shown in Appendix $\mathrm{H}$. The theoretical and simulated near-field distributions are in excellent agreement. In symmetric communication, we assumed equal input power 1:1 for both focal points, whereas in asymmetric communication, the power ratio is $2: 3$. By utilizing mathematical calculation based on demonstrated equations, we form weight factors $a_{1}$ $=a_{2}=0.5$ for symmetric focal points and $a_{1}=0.25, a_{2}$ $=0.75$ for asymmetric focal points. Also, Figure 6 presents the digital schemes, including amplitude and phase, which are applied to the designed metasurface to send the specified binary information through two channels. When the symbol 11 is sent across the channel, the total efficiency is calculated as $61.76 \%$ for the symmetric case, whereas total efficiency in the asymmetric case is calculated as $63.83 \%$. We define the focusing efficiency as the fraction of the incident beam reflected from meta-lens and concentrated in the plane of focus with a radius equal to three times the full width at half maximum (FWHM) spot size [46], [47]. A comprehensive study to define and calculate the different types of efficiency is provided in Appendix I. It is seen that the amplitude and phase metasurface can perform near-field modulations over both space and time domains to transmit the information via multiple dynamic channels independently. Note that this binary data transmission approach is extendable to ternary or higher bit communication in order to increase the transmission capacity in the channel [48] or nonreciprocal communication [49].

\section{B. An Application to 3D Wireless Agile Interconnects}

Three-dimensional integrated circuits (3D-IC) consist of the stacking of multiple layers of active devices or complete chips interconnected by a very dense array of vertical interconnects called through-silicon vias (TSVs). Such an integration technology has become commonplace in modern multiprocessor systems, mainly due to its superior noise immunity, higher device density, lower power, and potential for the tight interconnection of heterogeneous technologies [50]. Relevant examples include High-Bandwidth Memory (HBM) modules [51], scalable multicore processors [52], processor-in-memory solutions [53], and ultra-efficient heterogeneous computing systems with sensing capabilities [54].

A key aspect of 3D-ICs is the dense vertical interconnect. Given the relatively low distance between layers and the potentially tiny pitch of TSVs, 3D Networks-on-Chips (3D NoCs) have the potential energy efficiency and bandwidth density to satisfy the increasing data transfer requirements of massive multiprocessor systems [54], [55]. On the downside, $3 \mathrm{D}$ NoCs are prone to failures due to thermal problems or technological aspects derived from the small pitch and precise alignment requirements of the vias [56]. These reliability issues can be alleviated thanks to high-speed wireless nearfield capacitive or inductive links as proposed by various groups [57], [58]. However, both vias or near-field wireless interconnects can only carry a message at a time and transport them immediately above or below, which poses a fundamental performance and efficiency bottleneck because messages need to be decoded and forwarded by multiple intermediate routers.

The multi-focal meta-lens approach presented in this paper has the potential of removing these bottlenecks in several ways, as illustrated in Figure 7. Each processor or the processor group may be equipped with an antenna and a transmissive meta-lens, complementing or replacing the existing 3D NoC. Depending on the data transfer needs, the transmitting metalens, TX in Figure 7, will create multiple channels pointing to the target receiving antennas in the near-field, RX1RX3 in Figure 7. If the message needs to reach an antenna outside of the near-field of the transmitter, the transmission can be delivered to an intermediate node in the near-field following a shortest-path routing [56] or directly through farfield transmission. To that end, the transmissive metasurface could be reprogrammed to provide beam steering capabilities 
instead of focusing. By allowing channels to be created in any spot of the near-field sphere, the meta-lens allows us to bypass any intermediate router along the vertical or horizontal paths, reducing the latency and energy of communication and also allowing for rerouting messages very easily if one of the intermediate wired links fails. Moreover, the creation of multiple parallel channels has two important consequences: (1) it leads to efficient multicast, this is, the delivery of the same message to multiple destinations, and (2) avoids vertical connections to become hotspots of congestion, thereby improving the overall throughput.

\section{CONCLUSION}

In summary, we suggested a reprogrammable meta-device with amplitude and phase control at $\mathrm{THz}$ frequencies by using digital graphene-based metasurface for the first time. Furthermore, the structure capability to generate tunable multiple focal points in the half-space with an adjustable power ratio has been investigated. Also, this meta-device can control the amplitude of redirected energy by engineering the unit cell's amplitude. The simulation results demonstrated an excellent agreement with the theoretical predictions in the various complex examples. The designed metasurface has been applied to realize a multi-channel direct transmission of information in the near-field for wireless communications in general and in a specific case for wireless agile chip-scale interconnects in particular. The digital message can be transmitted with fastchanging hardware codes on the programmable metasurface and, thereupon, control the external bias applied to graphene layers via an FPGA. This work features a paradigm for designing compact, re-writable, and multi-channel information broadcasting systems and may lead to a new frontier for highspeed information communications and signal processing.

\section{APPENDIX A}

\section{Circuit Model of Metasurface}

The employed equivalent circuit and utilized method are based on the model proposed in [34] and the equivalent circuit is illustrated in Figure 8. In addition, the optimized values for the components in the equivalent circuit are summarized in Table I.

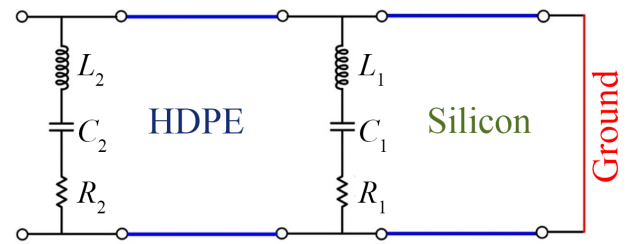

Fig. 8. The employed equivalent circuit model for calculating the reflection spectra of the employed metasurface.

\section{APPENDIX B}

\section{VOLTAGE FOR BIASING}

The required DC voltage versus chemical potential is illustrated in Figure 9. According to Figure 9, we calculated the necessary voltage for each digital state in Table II.

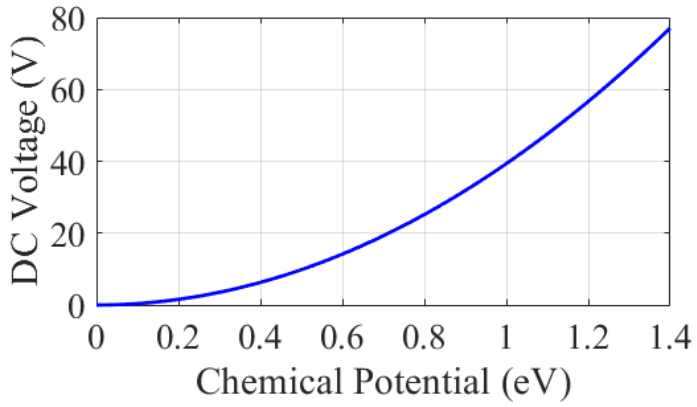

Fig. 9. Required DC voltage for biasing graphene layers.

\section{APPENDIX C \\ POTENTIAL FABRICATION METHOD}

Fabrication of the metasurface can be prepared with a lithography technique. Moreover, we should be able to bias the graphene patches through an external electric field for tuning. In this way, the graphene chemical potential is manipulated by changing the DC bias voltage magnitude. The capacitive structure is utilized in the suggested unit cell to tune the charge density of hBN-sandwiched graphene layers. The biasing panel is composed of polycrystalline silicon, Al2O3, and squareshaped monolayer graphene with a predetermined size [33]. The thickness of these auxiliary layers is much smaller than the wavelength in operating frequency, so their impact on the reflection amplitude and phase is negligible. The process of fabrication may follow the steps below [34]. In the first step, using a spin-coating solution, the silicon layer can be coated on a silicon wafer. The graphene monolayer can be fabricated using CVD graphene grown on $\mathrm{Cu}$ foil and transferred onto a spacer of $65 \mathrm{~nm}$-thick $\mathrm{Al} 2 \mathrm{O} 3$ layer that was uniformly deposited by the atomic layer deposition on an ultrahigh resistivity p-type polycrystalline wafer [59]. After being transferred to wafers, the excess graphene was removed in the sheets using $100 \mathrm{keV}$ electron beam lithography in PMMA, followed by an oxygen plasma etch, leaving only the squareshaped regions with a predetermined size. Then, the HDPE substrate used as a supporting layer during the transfer process is kept on top of the graphene to act as a dielectric between two graphene patches. The second graphene monolayer is transferred onto the top of the $\mathrm{Al} 2 \mathrm{O} 3$ layer. Eventually, the bottom silicon wafer can be peeled off using a KHF solution with metal deposited on the silicon layer's back as a ground plane. In order to control the external bias, the metal electrodes are patterned by optical lithography followed by a deposition of $5 \mathrm{~nm}$ of chromium on top of the graphene layers, $50 \mathrm{~nm}$ of gold, and a lift-off process. By employing this multilayer graphene-based structure, the stacked structure can avoid the potentially limited amplitude and phase modulation of singlelayer graphene [34]. Therefore, each layer can efficiently offer a broader amplitude and phase tuning range.

\section{APPENDIX D MOdUlation SPEED}

As the most significant advantage, graphene is a promising candidate for creating a reconfigurable meta-device since its conductivity can be tuned by shifting the Fermi energy level 
TABLE I

THE VALUES OF THE CIRCUIT COMPONENTS FOR THE EMPLOYED EQUIVALENT CIRCUIT MODEL.

\begin{tabular}{|l|l|l|l|l|l|l|l|l|l|l|l|}
\hline $\begin{array}{l}\text { Code } \\
\text { Amp }\end{array}$ & $\begin{array}{l}\text { Code } \\
\text { Phase }\end{array}$ & $\mathbf{S i m . A m p . S i m . ~}$ & $\mathbf{C} . \mathbf{M}$. & $\begin{array}{l}\text { C. } \\
\text { Phase }\end{array}$ & $\boldsymbol{R}_{1}(\boldsymbol{\Omega})$ & $\boldsymbol{L}_{1}(\mathbf{p H})$ & $\boldsymbol{C}_{1}(\mathbf{f F})$ & $\boldsymbol{R}_{2}(\boldsymbol{\Omega})$ & $\boldsymbol{L}_{2}(\mathbf{p H})$ & $\boldsymbol{C}_{2}(\mathbf{f F})$ \\
\hline 0 & 0 & 0.13 & 76 & 0.13005 & 76.0016 & 1228.70 & 16.5005 & 148.651 & 560.346 & 10.301457 & 87.3308 \\
\hline 0 & 1 & 0.2 & 112 & 0.19974 & 111.918 & 1046.44 & 159.371 & 519.757 & 351.018 & 7.0476625 & 243.039 \\
\hline 0 & 2 & 0.2 & 241 & 0.19895 & 241.001 & 116.359 & 6.18131 & 0.04160 & 320.666 & 0.7424282 & 1 \\
\hline 0 & 3 & 0.13 & 350 & 0.12999 & 350.010 & 1021.44 & 1.51574 & 0.40338 & 863.768 & 2.2952483 & 1.02573 \\
\hline 1 & 0 & 0.42 & 47 & 0.41998 & 46.9982 & 1497.69 & 243.996 & 1453.03 & 680.983 & 29.558767 & 63.8721 \\
\hline 1 & 1 & 0.46 & 115 & 0.45929 & 114.916 & 824.046 & 138.374 & 319.525 & 227.948 & 16.918549 & 1159.01 \\
\hline 1 & 2 & 0.3 & 247 & 0.3 & 247.001 & 996.293 & 243.108 & 1.62759 & 233.342 & 1.9189604 & 0.39784 \\
\hline 1 & 3 & 0.36 & 342 & 0.29656 & 342.546 & 1210.21 & 432.711 & 715.864 & 618.340 & 0.25 & 0.33894 \\
\hline 2 & 0 & 0.67 & 48 & 0.66999 & 47.9996 & 569.481 & 55.1914 & 319.499 & 434.941 & 291.13989 & 269.716 \\
\hline 2 & 1 & 0.67 & 122 & 0.67098 & 121.566 & 545.268 & 42.3961 & 929.638 & 105.091 & 22.037014 & 310.806 \\
\hline 2 & 2 & 0.63 & 241 & 0.62951 & 237.378 & 0.125 & 687.488 & 962.293 & 102.886 & 0.0161094 & 0.41559 \\
\hline 2 & 3 & 0.64 & 313 & 0.63969 & 312.152 & 571.402 & 348.706 & 186.147 & 280.526 & 7.3524624 & 0.11730 \\
\hline 3 & 0 & 0.82 & 22 & 0.82 & 22 & 1292.94 & 130.858 & 394.506 & 1255.67 & 862.31739 & 655.990 \\
\hline 3 & 1 & 0.81 & 136 & 0.81005 & 135.993 & 38.5131 & 10.8429 & 139.067 & 316.364 & 38.205104 & 35.9461 \\
\hline 3 & 2 & 0.81 & 253 & 0.81 & 253 & 27.4297 & 0 & 0.33230 & 0 & 78.569106 & 0.25 \\
\hline 3 & 3 & 0.81 & 293 & 0.81 & 293 & 0.70154 & 52.4415 & 667.571 & 40.9159 & 0 & 0.25 \\
\hline
\end{tabular}

TABLE II

REQUIRED DC VOLTAGE FOR BIASING GRAPHENE PATCHES IN THE GRAPHENE-BASED UNIT CELL.

\begin{tabular}{|c|c|c|c|c|c|c|c|c|c|}
\hline \multirow{2}{*}{\multicolumn{2}{|c|}{$\begin{array}{l}\text { Phase Code } \\
\text { Amplitude Code }\end{array}$}} & 00 & 00 & 00 & 00 & 01 & 01 & 01 & 01 \\
\hline & & 00 & 01 & 10 & 11 & 00 & 01 & 10 & 11 \\
\hline $\begin{array}{l}\text { Chemical Potential } \\
(\mathrm{eV})\end{array}$ & Top & 0.7 & 1 & 0.1 & 0 & 0.7 & 0.9 & 0.3 & 0.1 \\
\hline Electric Field (V/nm) & & 0.2965 & 0.6052 & 0.0061 & 0 & 0.2965 & 0.4902 & 0.0545 & 0.0061 \\
\hline Voltage (V) & & 19.27 & 39.33 & 0.39 & 0 & 19.27 & 31.86 & 3.54 & 0.39 \\
\hline $\begin{array}{ll}\begin{array}{l}\text { Chemical } \\
(\mathrm{eV})\end{array} & \text { Potential } \\
\end{array}$ & Bottor & 1.2 & 1 & 0.3 & 0.2 & 1.25 & 1.25 & 0.5 & 1.1 \\
\hline Electric Field (V/nm) & & 0.8714 & 0.6052 & 0.0545 & 0.0242 & 0.9456 & 0.9456 & 0.1513 & 0.7323 \\
\hline Voltage (V) & & 56.64 & 39.33 & 3.54 & 1.57 & 61.45 & 61.45 & 9.83 & 47.59 \\
\hline \multicolumn{2}{|l|}{ Phase Code } & 10 & 10 & 10 & 10 & 11 & 11 & 11 & 11 \\
\hline \multicolumn{2}{|l|}{ Amplitude Code } & 00 & 01 & 10 & 11 & 00 & 01 & 10 & 11 \\
\hline $\begin{array}{l}\text { Chemical Potential } \\
(\mathrm{eV})\end{array}$ & Top & 0.6 & 0.6 & 0.5 & 0.7 & 0.7 & 0.8 & 1 & 1.25 \\
\hline Electric Field (V/nm) & & 0.2179 & 0.2179 & 0.1513 & 0.2965 & 0.2965 & 0.3873 & 0.6052 & 0.9456 \\
\hline Voltage $(\mathrm{V})$ & & 14.16 & 14.16 & 9.83 & 19.27 & 19.27 & 25.17 & 39.33 & 61.45 \\
\hline $\begin{array}{ll}\begin{array}{l}\text { Chemical } \\
(\mathrm{eV})\end{array} & \text { Potential } \\
\end{array}$ & Bottor & n 1.1 & 1 & 0.1 & 0 & 1.1 & 0.9 & 0.6 & 0.2 \\
\hline Electric Field (V/nm) & & 0.7323 & 0.6052 & 0.0061 & 0 & 0.7323 & 0.4902 & 0.2179 & 0.0242 \\
\hline Voltage (V) & & 47.59 & 39.33 & 0.39 & 0 & 47.59 & 31.86 & 14.16 & 1.57 \\
\hline
\end{tabular}

through chemical doping or external electrostatic gating. The required electrostatic voltage bias can be applied to each graphene-based digital element through a gold/chromium electrical contact where Au contact touches the silicon layer, and the $\mathrm{Au} / \mathrm{Cr}$ contact is connected to the graphene metasurface [60]. In the designed meta-device, an FPGA can be easily used to produce the desired electrostatic voltages at its output pins and provide the required voltage for the digital sequence [61]. Accordingly, a different digital sequence can be transmitted through a real-time switching between the arbitrary digital layout of the reflection amplitude and phase, which has already been registered into the internal memory of FPGA. The switching speed depends on various parameters, such as the time constant (RC) of the biasing circuit. Reducing the graphene/metal contact resistance and decreasing capacitance enables the digital metasurface to be immediately programmed with a noticeable speed of over $\mathrm{GHz}$ [60]. The metasurface's contact resistance can be reduced by adjusting the graphene/metal contact as long as it does not perturb the digital metasurfaces' reflection behavior. Besides, the capacitance can be decreased by increasing the $\mathrm{Al} 2 \mathrm{O} 3$ layer thickness. However, this method will decrease the carrier concentration in the auxiliary layer and enhances the required voltage. Thus, designers should evaluate a proper value for parameters to achieve a high-speed switching between each digital element's different operational statuses, yielding realtime near-field manipulation. It should be regarded that the switching speed between different information packets in digital metasurface determines by the operating frequency of FPGA.

\section{APPENDIX E}

\section{EleCtric Field Distribution for Bifocal ExAMPLES}

The one-dimensional electric field distribution for the second example is illustrated in Figure 10. Moreover, the onedimensional electric field distribution for the third example is shown in Figure 11. 


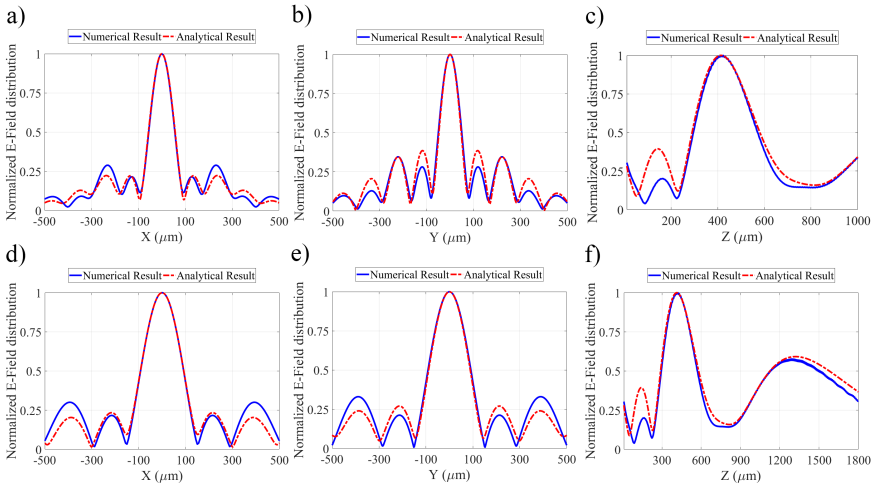

Fig. 10. (a)-(c) One-dimensional electric field distribution of graphenebased meta-lens for the first focal point in the second sample. (d)-(f) Onedimensional electric field distribution of graphene-based meta-lens for the second focal point in the second sample. These figures contain the numerical and analytical results extracted from the full-wave simulations and the theoretical calculations.
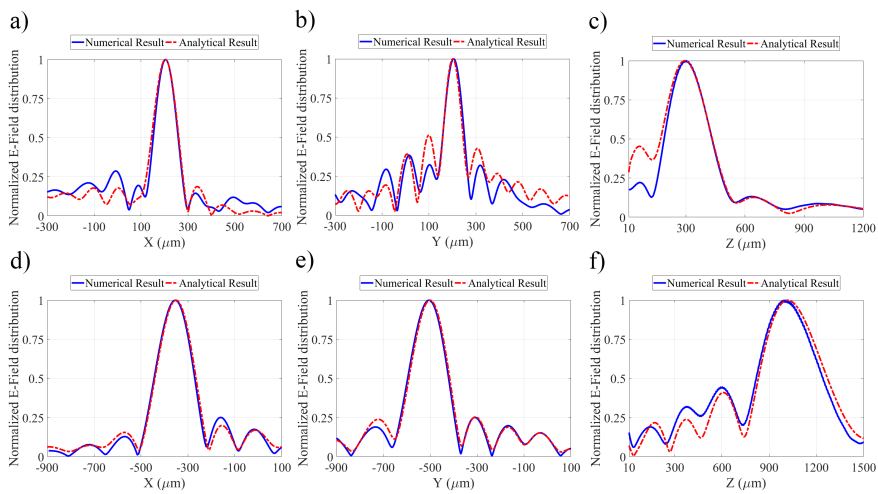

Fig. 11. (a)-(c) One-dimensional electric field distribution of graphenebased meta-lens for the first focal point in the third sample. (d)-(f) Onedimensional electric field distribution of graphene-based meta-lens for the second focal point in the third sample. These figures contain the numerical and analytical results extracted from the full-wave simulations and the theoretical calculations.

\section{APPENDIX F}

\section{POWER MODIFICATION}

The simulated electric field distribution for different weight factors is shown in Figure 12.

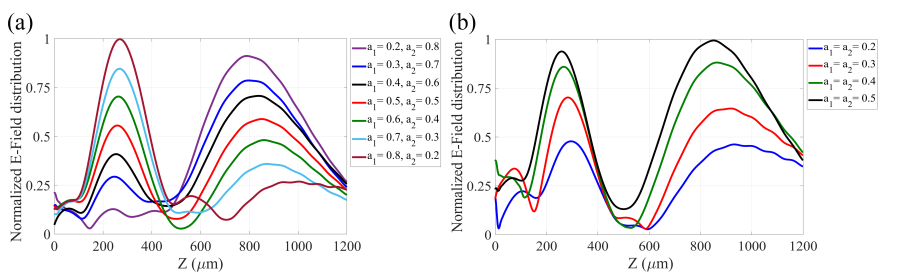

Fig. 12. (a) Electric field distribution of power modification examples with different weight factors. (b) Electric field distribution of power attenuation examples with various weight factors.

\section{APPENDIX G}

\section{ARbitrary Power Pattern Synthesis IN General} FORM

Two-dimensional power distributions at the focal planes for two arbitrary power pattern synthesis example is illustrated in Figures 13 and 14.
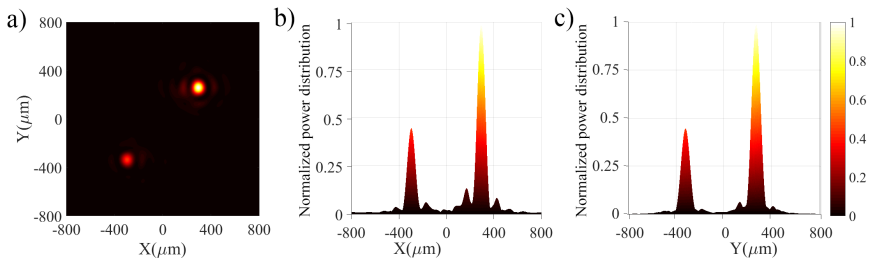

Fig. 13. (a) Two-dimensional power distributions at the focal plane for the first case. (b), (c) Side view of power distributions in this example. All numerical results are extracted from a full-wave simulation.
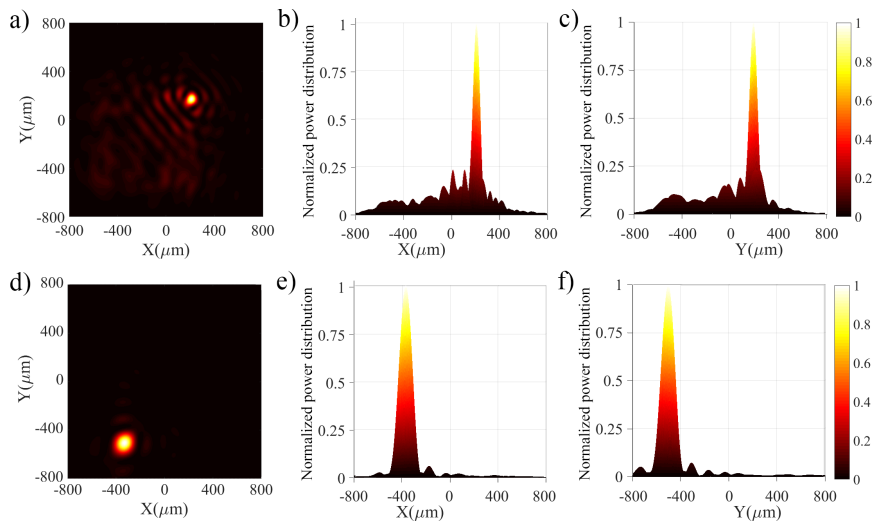

Fig. 14. (a) Two-dimensional power distributions at the first focal plane for the second case. (b), (c) Side view of power distributions in this example. (d) Two-dimensional power distributions at the second focal plane for the second case. (e), (f) Side view of power distributions in this example. All numerical results are extracted from a full-wave simulation.

\section{APPENDIX H}

\section{ReProgrammable Multi-Channel THz COMMUNICATIONS}

Digital profile and two-dimensional power distribution on the focal plane for the symmetric communication system are shown in Figures 15 and 16. Also, digital profile and twodimensional power distribution on the focal plane for the asymmetric communication system are shown in Figures 17 and 18 . (a)

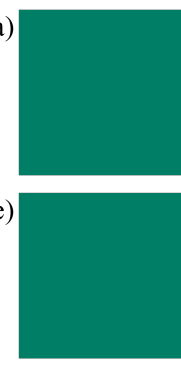

(i)

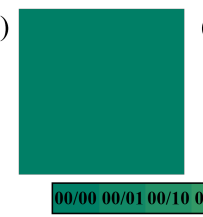

(b)

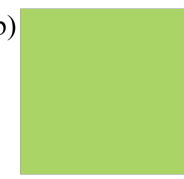

(f)

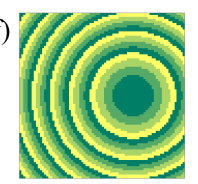

(c)

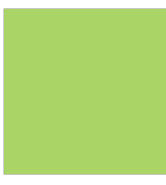

$(\mathrm{g})$

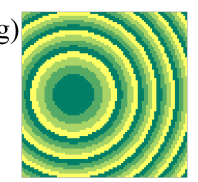

00011011

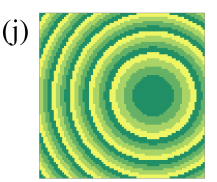

$(\mathrm{k})$

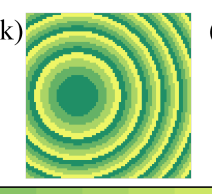

(1)
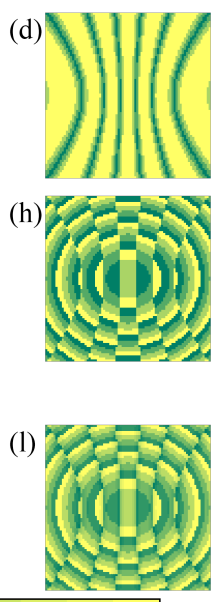

(h)

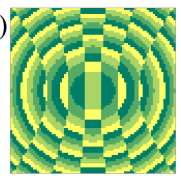

Fig. 15. The amplitude, phase, and digital profile of the multi-channel $\mathrm{THz}$ communication system in the symmetric scenario. (a)-(d) Amplitude, (e)-(h) Phase, and (i)-(1) Digital code. (Column 1) 00, (Column 2) 01, (Column 3) 10, and (Column 4) 11. 
TABLE III

RESOLUTION EFFICIENCY OF THE FOCAL POINTS IN SYMMETRIC AND ASYMMETRIC COMMUNICATIONS.

\begin{tabular}{|l|l|l|l|l|l|l|}
\hline Transmitted Code & \multicolumn{2}{l|}{$\begin{array}{l}\text { First Focal Point for symbol } \\
11\end{array}$} & \multicolumn{2}{l|}{$\begin{array}{l}\text { Second Focal Point for symbol } \\
11\end{array}$} \\
\hline & $2 w$ & $2 w_{0}$ & $\eta_{\text {resolution }}$ & $2 w$ & $2 w_{0}$ & $\eta_{\text {resolution }}$ \\
\hline Symmetric (Phase only) & 90 & 47.15 & 0.52 & 90 & 47.15 & 0.52 \\
\hline $\begin{array}{l}\text { Symmetric (Amplitude and } \\
\text { Phase) }\end{array}$ & 84 & 47.15 & 0.56 & 84 & 47.15 & 0.56 \\
\hline Asymmetric (Phase only) & 88.5 & 32.85 & 0.37 & 112.5 & 75.86 & 0.67 \\
\hline $\begin{array}{l}\text { Asymmetric (Amplitude and } \\
\text { Phase) }\end{array}$ & 80 & 32.85 & 0.41 & 108 & 75.86 & 0.70 \\
\hline
\end{tabular}
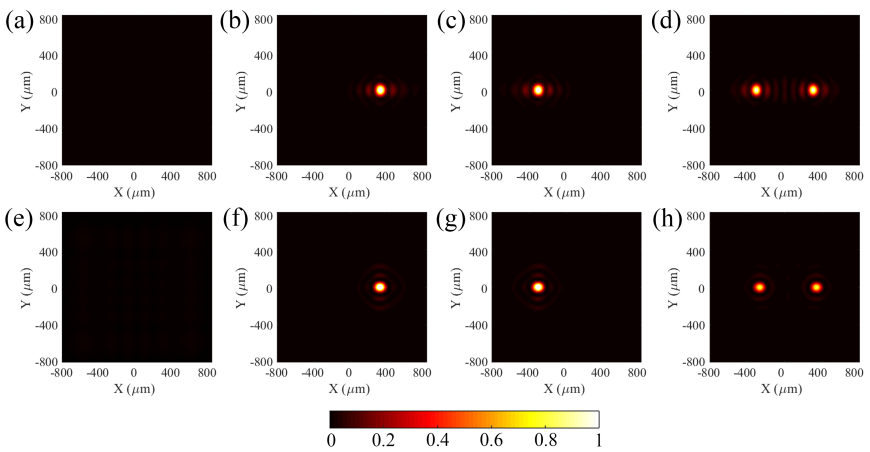

Fig. 16. Power distributions in multi-channel $\mathrm{THz}$ communication system in the symmetric scenario. (a)-(d) Analytical, and (e)-(h) Numerical results. (Column 1) 00, (Column 2) 01, (Column 3) 10, and (Column 4) 11. (a)

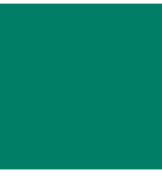

(e)

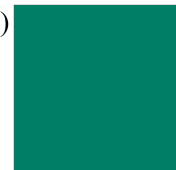

(b)

(f)

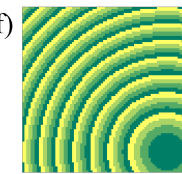

(c)

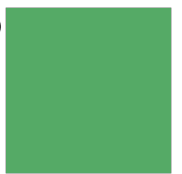

(g)

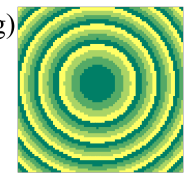

(h)
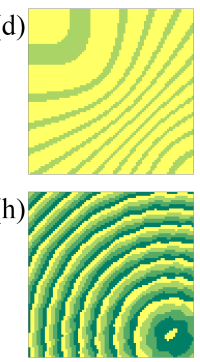

0011011

(i)

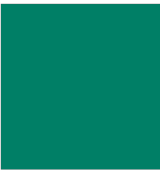

(j)

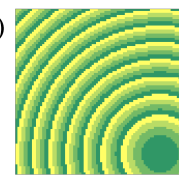

(k)

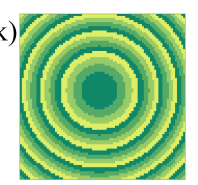

(1)

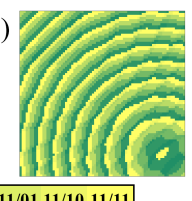

$00 / 00$ 00/01 00/10 00/11 01/00 01/01 01/10 01/11 10/00 10/01 10/10 10/11 11/00 11/01 11/10 11/11

Fig. 17. The amplitude, phase, and digital profile of the multi-channel $\mathrm{THz}$ communication system in the asymmetric scenario. (a)-(d) Amplitude, (e)-(h) Phase, and (i)-(1) Digital code. (Column 1) 00, (Column 2) 01, (Column 3) 10, and (Column 4) 11

\section{APPENDIX I \\ EFFICIENCY ANALYSIS}

The efficiency in the meta-lens can be examined from three different viewpoints. We study these three viewpoints in the following:

Unit cell level efficiency: At this level, we analyze the reflection efficiency of unit cells in the metalens. So, we can define unit cell efficiency for each case as

$$
\eta_{\text {unit cell }}=\frac{\sum_{i=1}^{M=80} \sum_{j=1}^{M=80} a_{i j}}{M^{2}}
$$

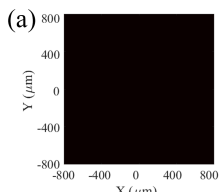

(e)

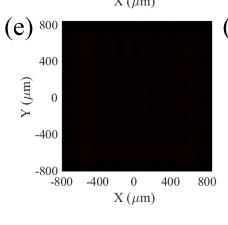

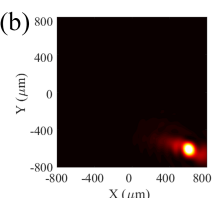

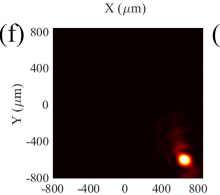

$\mathrm{x}(\mu \mathrm{m})$

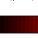

$+$
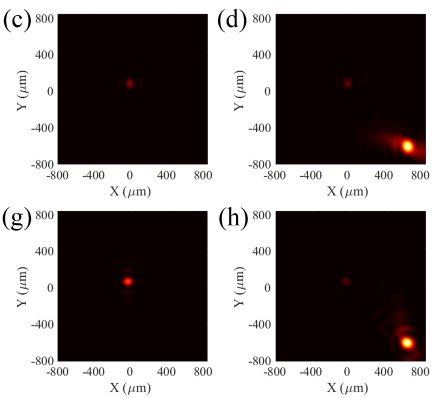

Fig. 18. Power distributions in multi-channel $\mathrm{THz}$ communication system in the asymmetric scenario. (a)-(d) Analytical, and (e)-(h) Numerical results. (Column 1) 00, (Column 2) 01, (Column 3) 10, and (Column 4) 11.

where $\eta_{\text {unitcell }}$ is unit cell level efficiency, $M$ is number of unit cells in $x$ - and $y$-direction, and $a_{i j}$ is unit cell amplitude. In the presented examples for symmetric and asymmetric communication, we assumed $M=80$. According to the given formulation for unit cell efficiency, this value is reported as $61.25 \%$ and $73.98 \%$ for symmetric and symmetric communication, respectively. The higher efficiency is obtained when we used asymmetric points with different weight factors for the communication. Therefore, we can increase the system's efficiency by selecting the proper value for focal points and weight factors.

Focusing efficiency: The ratio of the concentrated energy on the focal point region to the incident energy is the most important parameter in the meta-lens. According to the definition, we can define it as below,

$$
\eta_{\text {focusing }}=\frac{\int_{-3 r_{F W H M}}^{3 r_{F W H M}} \int_{0}^{2 \pi}|E|^{2} d \varphi d r}{\int_{-0.5 x_{\max }}^{0.5 x_{\max }} \int_{-0.5 y_{\max }}^{0.5 y_{\max }}\left|E_{\text {inc }}\right|^{2} d x d y}
$$

In the above equation $\eta_{\text {focusing }}$ is focusing efficiency, $r_{F W H M}$ refers to the limit of the full width at half maximum (FWHM), $E$ indicates the electric field in the focal plane, $x_{\max }=M \times u$ and $y_{\max }=M \times u$ are the dimensions of the metasurface and $E_{i n c}$ is the electric field of the incident beam. Indeed, the focusing efficiency is defined as the ratio of the energy in three times the FWHM range with the focal point as the center (circle of radius three times FWHM) to the total incident energy [46], [62]-[65].

The focusing efficiency can only be defined when the metalens has generated the focal point. So, when we send the symbol "00" to the channel, the focusing efficiency can not be 
defined. For the symbol " 01 " and "10", the focusing efficiency can be calculated as the lens with a single focal point. Finally, in the case of transmitting " 11 " in the channel, total efficiency is defined as a summation of the focusing efficiency of both focal points. It is clear that for symmetric symbols, the incident energy is divided among symmetric focal points, and the focusing efficiency of every single focal point theoretically will not exceed $50 \%$ [66], [67]. When the symbol "11" is sent across the channel, the total efficiency is calculated as $61.76 \%$ for the symmetric case, whereas total efficiency in the asymmetric case is calculated as $63.83 \%$.

Resolution efficiency: Metasurfaces with only phase control can also concentrate the wave on arbitrary multifocal points. Nevertheless, controlling the amplitude lead us to a narrower beam which can provide an efficient communication system. Also, the narrower beam is a demanding feature in imaging systems. In order to examine the focusing width, we define $2 w$ as the full width at half maximum, which is the function of the focal point and the aperture size of the metasurface. On the other hand, the diffraction-limited full-beam waist is given by

$$
2 w_{0}=1.22 \frac{\lambda_{0} R_{f}}{D}
$$

In this equation $\lambda_{0}$ is wavelength in operating frequency, $R_{f}=\sqrt{x_{f}^{2}+y_{f}^{2}+z_{f}^{2}}$ is the focal distance from the center of meta-lens, and the aperture size of the metasurface is indicated by $D$. In order to compare the resolution efficiency of the meta-lens in different cases, we define resolution efficiency as $\eta_{\text {resolution }}=2 w_{0} /(2 w)$ [44]. The higher value of resolution efficiency shows that we are closer to the diffraction limit. Table III shows the calculated resolution efficiency for symmetric and asymmetric communication. According to obtained result, in both symmetric and asymmetric communication, the resolution efficiency is increased when we used both amplitude and phase to manipulate the reflected wave. Hence, amplitude control plays a vital role in controlling the beamwidth.

\section{ACKNOWLEDGMENT}

This work has been supported by Home of the $5 \mathrm{G}$ and 6G Innovation Centres. S. Abadal acknowledges support from the European Unions Horizon 2020 research and innovation programme under grant agreement No 863337.

\section{REFERENCES}

[1] N. Yu and F. Capasso, "Optical metasurfaces and prospect of their applications including fiber optics," Journal of Lightwave Technology, vol. 33, no. 12, pp. 2344-2358, 2015.

[2] T. J. Cui, "Microwave metamaterials," National Science Review, vol. 5, no. 2, pp. 134-136, 2018.

[3] Y. Liang, Q. Tan, W. Zhou, X. Zhou, Z. Wang, G. Zhou, and X. Huang, "Refractive index sensing utilizing tunable polarization conversion efficiency with dielectric metasurface," Journal of Lightwave Technology, vol. 39, no. 2, pp. 682-687, 2020.

[4] Y. Zhang, C.-P. Huang, and H.-c. Chang, "Super diffraction in a singlelayer metasurface," Journal of Lightwave Technology, vol. 34, no. 14 , pp. 3312-3316, 2016.

[5] P. Salami and L. Yousefi, "Far-field subwavelength imaging using phase gradient metasurfaces," Journal of Lightwave Technology, vol. 37, no. 10 , pp. 2317-2323, 2019.
[6] S. Fallah, K. Rouhi, and A. Abdolali, "Optimized chemical potential graphene-based coding metasurface approach for dynamic manipulation of terahertz wavefront," Journal of Physics D: Applied Physics, vol. 53, no. 8, p. 085102, 2019.

[7] A. Babaee, A. Momeni, A. Abdolali, and R. Fleury, "Parallel analog computing based on a $2 \times 2$ multiple-input multiple-output metasurface processor with asymmetric response," Physical Review Applied, vol. 15, no. 4, p. $044015,2021$.

[8] Q. He, S. Sun, and L. Zhou, "Tunable/reconfigurable metasurfaces: physics and applications," Research, vol. 2019, 2019.

[9] M. Kiani, M. Tayarani, A. Momeni, H. Rajabalipanah, and A. Abdolali, "Self-biased tri-state power-multiplexed digital metasurface operating at microwave frequencies," Optics Express, vol. 28, no. 4, pp. 5410-5422, 2020.

[10] S. E. Hosseininejad, K. Rouhi, M. Neshat, A. Cabellos-Aparicio, S. Abadal, and E. Alarcón, "Digital metasurface based on graphene: an application to beam steering in terahertz plasmonic antennas," IEEE Transactions on Nanotechnology, vol. 18, pp. 734-746, 2019.

[11] Z. Han, K. Kohno, H. Fujita, K. Hirakawa, and H. Toshiyoshi, "Tunable terahertz filter and modulator based on electrostatic mems reconfigurable srr array," IEEE Journal of Selected Topics in Quantum Electronics, vol. 21, no. 4, pp. 114-122, 2014.

[12] R. Kargar, K. Rouhi, and A. Abdolali, "Reprogrammable multifocal thz metalens based on metal-insulator transition of vo2-assisted digital metasurface," Optics Communications, vol. 462, p. 125331, 2020.

[13] X. Zhao, K. Fan, J. Zhang, H. R. Seren, G. D. Metcalfe, M. Wraback, R. D. Averitt, and X. Zhang, "Optically tunable metamaterial perfect absorber on highly flexible substrate," Sensors and Actuators A: Physical, vol. 231, pp. 74-80, 2015.

[14] T. J. Cui, M. Q. Qi, X. Wan, J. Zhao, and Q. Cheng, "Coding metamaterials, digital metamaterials and programmable metamaterials," Light: Science \& Applications, vol. 3, no. 10, pp. e218-e218, 2014.

[15] R. Y. Wu, C. B. Shi, S. Liu, W. Wu, and T. J. Cui, "Addition theorem for digital coding metamaterials," Advanced Optical Materials, vol. 6, no. 5, p. 1701236, 2018.

[16] S. E. Hosseininejad, N. Komjani, and M. T. Noghani, "A comparison of graphene and noble metals as conductors for plasmonic one-dimensional waveguides," IEEE Transactions on Nanotechnology, vol. 14, no. 5, pp. 829-836, 2015.

[17] P. Tassin, T. Koschny, and C. M. Soukoulis, "Graphene for terahertz applications," Science, vol. 341, no. 6146, pp. 620-621, 2013.

[18] N. K. Emani, D. Wang, T.-F. Chung, L. J. Prokopeva, A. V. Kildishev, V. M. Shalaev, Y. P. Chen, and A. Boltasseva, "Plasmon resonance in multilayer graphene nanoribbons," Laser \& Photonics Reviews, vol. 9, no. 6, pp. 650-655, 2015.

[19] P. C. Wu, N. Papasimakis, and D. P. Tsai, "Self-affine graphene metasurfaces for tunable broadband absorption," Physical Review Applied, vol. 6, no. 4, p. 044019, 2016.

[20] M. Rahmanzadeh, H. Rajabalipanah, and A. Abdolali, "Analytical investigation of ultrabroadband plasma-graphene radar absorbing structures," IEEE Transactions on Plasma Science, vol. 45, no. 6, pp. 945-954, 2017.

[21] M. Amin, O. Siddiqui, and M. Farhat, "Polarization-state modulation in fano resonant graphene metasurface reflector," Journal of Lightwave Technology, 2021.

[22] S. K. Patel, J. Parmar, V. Sorathiya, R. Zakaria, V. Dhasarathan, and T. K. Nguyen, "Graphene-based plasmonic absorber for biosensing applications using gold split ring resonator metasurfaces," Journal of Lightwave Technology, 2021.

[23] D.-P. Tsai, "Exploring the electromagnetic information of metasurfaces," National Science Review, vol. 7, no. 12, pp. 1845-1846, 2020.

[24] S. Abadal, T.-J. Cui, T. Low, and J. Georgiou, "Programmable metamaterials for software-defined electromagnetic control: Circuits, systems, and architectures," IEEE Journal on Emerging and Selected Topics in Circuits and Systems, vol. 10, no. 1, pp. 6-19, 2020.

[25] H. Li, G. Wang, T. Cai, H. Hou, and W. Guo, "Wideband transparent beam-forming metadevice with amplitude-and phase-controlled metasurface," Physical Review Applied, vol. 11, no. 1, p. 014043, 2019.

[26] L. Li, T. J. Cui, W. Ji, S. Liu, J. Ding, X. Wan, Y. B. Li, M. Jiang, C.-W. Qiu, and S. Zhang, "Electromagnetic reprogrammable codingmetasurface holograms," Nature communications, vol. 8, no. 1, pp. 1-7, 2017.

[27] X. Wan, Q. Zhang, T. Y. Chen, L. Zhang, W. Xu, H. Huang, C. K. Xiao, Q. Xiao, and T. J. Cui, "Multichannel direct transmissions of near-field information," Light: Science \& Applications, vol. 8, no. 1, pp. 1-8, 2019. 
[28] T. J. Cui, S. Liu, G. D. Bai, Q. Ma et al., "Direct transmission of digital message via programmable coding metasurface," Research, vol. 2019 , p. 2584509,2019

[29] S. E. Hosseininejad, E. Alarcón, N. Komjani, S. Abadal, M. C. Lemme, P. H. Bolívar, and A. Cabellos-Aparicio, "Study of hybrid and pure plasmonic terahertz antennas based on graphene guided-wave structures," Nano communication networks, vol. 12, pp. 34-42, 2017.

[30] M. Rahmanzadeh, B. Rejaei, M. Memarian, and A. Khavasi, "Analytical and rigorous method for analysis of an array of magnetically-biased graphene ribbons," Optics express, vol. 27, no. 20, pp. 28 395-28 409, 2019.

[31] G. W. Hanson, "Dyadic green's functions for an anisotropic, nonlocal model of biased graphene," IEEE Transactions on antennas and propagation, vol. 56, no. 3, pp. 747-757, 2008.

[32] A. Momeni, K. Rouhi, H. Rajabalipanah, and A. Abdolali, "An information theory-inspired strategy for design of re-programmable encrypted graphene-based coding metasurfaces at terahertz frequencies," Scientific reports, vol. 8, no. 1, pp. 1-13, 2018.

[33] K. Rouhi, H. Rajabalipanah, and A. Abdolali, "Real-time and broadband terahertz wave scattering manipulation via polarization-insensitive conformal graphene-based coding metasurfaces," Annalen der Physik, vol. 530 , no. 4, p. 1700310,2018

[34] K. Rouhi, H. Rajabalipanah, and A. Abdolali, "Multi-bit graphene-based bias-encoded metasurfaces for real-time terahertz wavefront shaping: From controllable orbital angular momentum generation toward arbitrary beam tailoring," Carbon, vol. 149, pp. 125-138, 2019.

[35] M. Jiang, F. Hu, L. Zhang, B. Quan, W. Xu, H. Du, D. Xie, and Y. Chen, "Electrically triggered vo2 reconfigurable metasurface for amplitude and phase modulation of terahertz wave," Journal of Lightwave Technology, 2021.

[36] J. Zhao, J. Song, T. Xu, T. Yang, and J. Zhou, "Controllable linear asymmetric transmission and perfect polarization conversion in a terahertz hybrid metal-graphene metasurface," Optics express, vol. 27, no. 7, pp. 9773-9781, 2019.

[37] T. Yatooshi, A. Ishikawa, and K. Tsuruta, "Terahertz wavefront control by tunable metasurface made of graphene ribbons," Applied Physics Letters, vol. 107, no. 5, p. 053105, 2015.

[38] L. Falkovsky, "Unusual field and temperature dependence of the hall effect in graphene," Physical Review B, vol. 75, no. 3, p. 033409, 2007.

[39] F. Wang, Y. Zhang, C. Tian, C. Girit, A. Zettl, M. Crommie, and Y. R. Shen, "Gate-variable optical transitions in graphene," science, vol. 320, no. 5873, pp. 206-209, 2008.

[40] L. Ren, Q. Zhang, J. Yao, Z. Sun, R. Kaneko, Z. Yan, S. Nanot, Z. Jin, I. Kawayama, M. Tonouchi et al., "Terahertz and infrared spectroscopy of gated large-area graphene," Nano Letters, vol. 12, no. 7, pp. 3711$3715,2012$.

[41] C. Hwang, D. A. Siegel, S.-K. Mo, W. Regan, A. Ismach, Y. Zhang, A. Zettl, and A. Lanzara, "Fermi velocity engineering in graphene by substrate modification," Scientific reports, vol. 2, p. 590, 2012.

[42] C. T. Phare, Y.-H. D. Lee, J. Cardenas, and M. Lipson, "Graphene electro-optic modulator with $30 \mathrm{ghz}$ bandwidth," Nature Photonics, vol. 9, no. 8, pp. 511-514, 2015.

[43] H. Rajabalipanah, K. Rouhi, A. Abdolali, S. Iqbal, L. Zhang, and S. Liu, "Real-time terahertz meta-cryptography using polarization-multiplexed graphene-based computer-generated holograms," Nanophotonics, vol. 9, no. 9 , pp. 2861-2877, 2020.

[44] S. E. Hosseininejad, K. Rouhi, M. Neshat, R. Faraji-Dana, A. CabellosAparicio, S. Abadal, and E. Alarcón, "Reprogrammable graphene-based metasurface mirror with adaptive focal point for thz imaging," Scientific reports, vol. 9, no. 1, pp. 1-9, 2019.

[45] X. Wang, J. Ding, B. Zheng, S. An, G. Zhai, and H. Zhang, "Simultaneous realization of anomalous reflection and transmission at two frequencies using bi-functional metasurfaces," Scientific reports, vol. 8 , no. 1 , pp. 1-8, 2018.

[46] A. Arbabi, Y. Horie, A. J. Ball, M. Bagheri, and A. Faraon, "Subwavelength-thick lenses with high numerical apertures and large efficiency based on high-contrast transmitarrays," Nature communications, vol. 6, no. 1, pp. 1-6, 2015.

[47] Y. Gu, R. Hao, and E.-P. Li, "Independent bifocal metalens design based on deep learning algebra," IEEE Photonics Technology Letters, vol. 33, no. 8, pp. 403-406, 2021

[48] R. Padovani, "Method and apparatus for providing ternary power control in a communication system," Jun. 25 2002, uS Patent 6,411,799.

[49] X. Chen, J. Zhang, C. Wen, K. Liu, Z. Zhu, S. Qin, and X. Yuan, "Optical nonlinearity and non-reciprocal transmission of graphene integrated metasurface," Carbon, vol. 173, pp. 126-134, 2021.
[50] A. W. Topol, D. C. La Tulipe, L. Shi, D. J. Frank, K. Bernstein, S. E. Steen, A. Kumar, G. U. Singco, A. M. Young, K. W. Guarini, and M. Ieong, "Three-dimensional integrated circuits," IBM Journal of Research and Development, vol. 50, no. 4, pp. 491-506, 2006.

[51] G. H. Loh, "3d-stacked memory architectures for multi-core processors," ACM SIGARCH computer architecture news, vol. 36, no. 3, pp. 453-464, 2008.

[52] N. Miura, Y. Koizumi, Y. Take, H. Matsutani, T. Kuroda, H. Amano, R. Sakamoto, M. Namiki, K. Usami, M. Kondo et al., "A scalable 3d heterogeneous multicore with an inductive thruchip interface," IEEE Micro, vol. 33, no. 6, pp. 6-15, 2013.

[53] D. Fick, R. G. Dreslinski, B. Giridhar, G. Kim, S. Seo, M. Fojtik, S. Satpathy, Y. Lee, D. Kim, N. Liu et al., "Centip3de: A cluster-based ntc architecture with 64 arm cortex-m3 cores in 3d stacked $130 \mathrm{~nm}$ cmos," IEEE Journal of Solid-State Circuits, vol. 48, no. 1, pp. 104$117,2012$.

[54] M. M. Shulaker, G. Hills, R. S. Park, R. T. Howe, K. Saraswat, P. Wong, and S. Mitra, "Three-dimensional integration of nanotechnologies for Computing and Data Storage on a Single Chip," Nature, vol. 547, no. 7661, pp. 74-78, 2017.

[55] B. S. Feero and P. P. Pande, "Networks-on-chip in a three-dimensional environment: A performance evaluation," IEEE Transactions on computers, vol. 58, no. 1, pp. 32-45, 2008.

[56] K. C. Chen, S. Y. Lin, H. S. Hung, and A. Y. A. Wu, "Topologyaware adaptive routing for nonstationary irregular mesh in throttled 3D NoC systems," IEEE Transactions on Parallel and Distributed Systems, vol. 24, no. 10, pp. 2109-2120, 2013.

[57] H. Ishikuro and T. Kuroda, "Wireless proximity interfaces with a pulsebased inductive coupling technique," IEEE Communications Magazine, vol. 48, no. 10, pp. 192-199, 2010.

[58] Y. Take, H. Matsutani, D. Sasaki, M. Koibuchi, T. Kuroda, and H. Amano, "3d noc with inductive-coupling links for building-block sips," IEEE Transactions on Computers, vol. 63, no. 3, pp. 748-763, 2012.

[59] Z. Geng, B. Hähnlein, R. Granzner, M. Auge, A. A. Lebedev, V. Y. Davydov, M. Kittler, J. Pezoldt, and F. Schwierz, "Graphene nanoribbons for electronic devices," Annalen der Physik, vol. 529, no. 11, p. 1700033, 2017.

[60] Y. Ding, X. Zhu, S. Xiao, H. Hu, L. H. Frandsen, N. A. Mortensen, and K. Yvind, "Effective electro-optical modulation with high extinction ratio by a graphene-silicon microring resonator," Nano letters, vol. 15 , no. 7, pp. 4393-4400, 2015.

[61] K. Rouhi, A. Abdolali, and S. Fallah, "Designing approach of terahertz broadband backscattering reduction based on combination of diffusion and absorption," Optik, vol. 246, p. 167771, 2021.

[62] W. Wang, Z. Guo, K. Zhou, Y. Sun, F. Shen, Y. Li, S. Qu, and S. Liu, "Polarization-independent longitudinal multi-focusing metalens," Optics express, vol. 23, no. 23, pp. 29855-29866, 2015.

[63] S. M. Kamali, E. Arbabi, A. Arbabi, and A. Faraon, "A review of dielectric optical metasurfaces for wavefront control," Nanophotonics, vol. 7, no. 6, pp. 1041-1068, 2018.

[64] B. Ratni, Z. Wang, K. Zhang, X. Ding, A. de Lustrac, G.-P. Piau, and S. N. Burokur, "Dynamically controlling spatial energy distribution with a holographic metamirror for adaptive focusing," Physical Review Applied, vol. 13, no. 3, p. 034006, 2020.

[65] X. Xiong, X. Wang, Z. Wang, Y. Gao, R. Peng, and M. Wang, "Constructing an achromatic polarization-dependent bifocal metalens with height-gradient metastructures," Optics Letters, vol. 46, no. 6, pp 1193-1196, 2021.

[66] X. Zang, H. Ding, Y. Intaravanne, L. Chen, Y. Peng, J. Xie, Q. Ke, A. V. Balakin, A. P. Shkurinov, X. Chen et al., "A multi-foci metalens with polarization-rotated focal points," Laser \& Photonics Reviews, vol. 13, no. 12, p. 1900182, 2019.

[67] S. Gao, C.-S. Park, C. Zhou, S.-S. Lee, and D.-Y. Choi, "Twofold polarization-selective all-dielectric trifoci metalens for linearly polarized visible light," Advanced Optical Materials, vol. 7, no. 21, p. 1900883 , 2019. 